\title{
Status and development of high-power laser facilities at the NLHPLP
}

Jianqiang $\mathrm{Zhu}^{1,2}$, Jian $\mathrm{Zhu}^{2,3}$, Xuechun $\mathrm{Li}^{1,2}$, Baoqiang $\mathrm{Zhu}^{1,2}$, Weixin $\mathrm{Ma}^{2,3}$, Xingqiang $\mathrm{Lu}^{1,2}$, Wei Fan ${ }^{1,2}$, Zhigang $\mathrm{Liu}^{1,2}$, Shenlei $\mathrm{Zhou}^{1,2}$, Guang $\mathrm{Xu}^{1,2}$, Guowen Zhang ${ }^{1,2}$, Xinglong Xie ${ }^{1,2}$, Lin Yang ${ }^{1,2}$, Jiangfeng Wang ${ }^{1,2}$, Xiaoping Ouyang ${ }^{1,2}$, Li Wang ${ }^{1,2}$, Dawei $\mathrm{Li}^{1,2}$, Pengqian Yang ${ }^{1,2}$, Quantang Fan ${ }^{1,2}$, Mingying Sun ${ }^{1,2}$, Chong $\mathrm{Liu}^{1,2}$, Dean $\mathrm{Liu}^{1,2}$, Yanli Zhang ${ }^{1,2}$, Hua Tao ${ }^{1,2}$, Meizhi Sun $^{1,2}$, Ping Zhu ${ }^{1,2}$, Bingyan Wang ${ }^{1,2}$, Zhaoyang Jiao ${ }^{1,2}$, Lei Ren ${ }^{1,2}$, Daizhong Liu $^{1,2}$, Xiang Jiao ${ }^{1,2}$, Hongbiao Huang ${ }^{1,2}$, and Zunqi Lin ${ }^{1,2}$

\footnotetext{
${ }^{1}$ National Laboratory on High Power Laser and Physics, Shanghai 201800, China

${ }^{2}$ Shanghai Institute of Optics and Fine Mechanics, Chinese Academy of Sciences, Shanghai 201800, China

${ }^{3}$ Shanghai Institute of Laser Plasma, Chinese Academy of Engineering and Physics, Shanghai 201800, China

(Received 28 March 2018; revised 7 August 2018; accepted 15 August 2018)
}

\begin{abstract}
In this paper, we review the status of the multifunctional experimental platform at the National Laboratory of High Power Laser and Physics (NLHPLP). The platform, including the SG-II laser facility, SG-II 9th beam, SG-II upgrade (SG-II UP) facility, and SG-II 5 PW facility, is operational and available for interested scientists studying inertial confinement fusion (ICF) and a broad range of high-energy-density physics. These facilities can provide important experimental capabilities by combining different pulse widths of nanosecond, picosecond, and femtosecond scales. In addition, the SG-II UP facility, consisting of a single petawatt system and an eight-beam nanosecond system, is introduced including several laser technologies that have been developed to ensure the performance of the facility. Recent developments of the SG-II 5 PW facility are also presented.
\end{abstract}

Keywords: high-power laser facility; inertial confinement fusion; solid-state amplifier

\section{Introduction}

Soon after the generation of the first laser in the 1960s, it was recognized that the energetic laser was one of the most promising and potent options to deliver focused power and energy required for inertial confinement fusion (ICF) in the laboratory ${ }^{[1-3]}$. Moreover, it was also crucial for highenergy-density (HED) scientific research and astrophysics. Therefore, high-power laser science and related technologies have gained impressive and successful improvements over the past several decades. A great number of facilities in many countries have been successfully established to study ICF and HED physics. Among these are the two most powerful facilities; the National Ignition Facility $(\mathrm{NIF})^{[4]}$, presently operated with 192 beamlines at the Lawrence Livermore National Laboratory (LLNL) in USA and the Laser Megajoule (LMJ) facility under construction in France ${ }^{[5]}$. Poten-

Correspondence to: J. Zhu and Y. Zhang, No. 390, Qinghe Road, Jiading District, Shanghai 201800, China. Email: jqzhu@siom.ac.cn (J. Zhu); zhangyl@siom.ac.cn (Y. Zhang). tially larger facilities may be involved to implement the ignition goals in the future ${ }^{[6,7]}$. In China, such research activities were initiated early and accordingly various facilities were built to greatly promote Chinese ICF studies ${ }^{[8,9]}$. As the major pioneer devoted to high-power laser technology and ICF research in China, the National Laboratory on High Power Laser and Physics (NLHPLP) and its precursor established a succession of facilities over almost 50 years as shown in Figure 1. Early on, a single $10^{10}-\mathrm{W}$ laser with a six-beam system was successfully developed in the 1970s. Afterward, a solid-state laser facility $\left(10^{12} \mathrm{~W}\right)$, originally called the No. 12 Facility, was proposed in 1980 and was fully operational in 1985. This facility was formally named the 'Shen Guang' facility in 1986, and distinguished from subsequent SG series facilities by being referred to as the SG-I facility. The SGI facility with two beams played an important role in the Chinese preliminary ICF research program ${ }^{[10-12]}$. Following greater requests for energy, wavelength, and pulse width from laser plasma interaction physics, the SG-II project was launched at the end of 1994, and in the same year, 


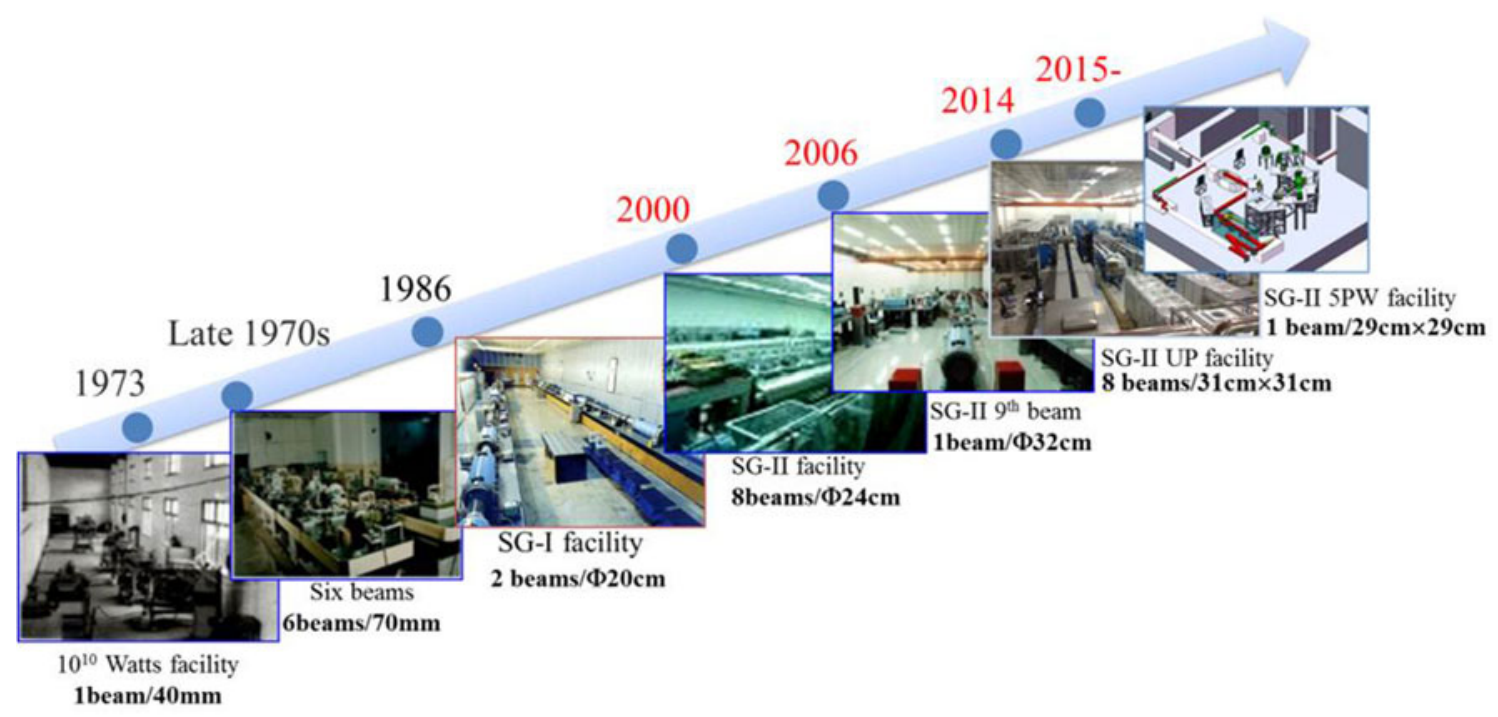

Figure 1. Photographs of a series of laser facilities built at the NLHPLP.

the SG-I facility was dismantled. Six years later, the SG-II facility ${ }^{[7,13-15]}$ with eight beams was put into normal operation serving various physical experiments. As the works continued, another new high-power laser, named the SG-II 9th beam with a larger diameter, was built and has been operated as a probing beam since 2006 . In order to satisfy the increasing demands of physical experiments, the fast ignition scheme for ICF studies, the SG-II upgrade (SG-II UP or SGII-U) program ${ }^{[16,17]}$ was approved, aiming at setting up a larger nanosecond output system with eight beams at $3 \omega$ $(0.351 \mu \mathrm{m})$ and kilojoule-class petawatt (PW) laser system based mainly on the SG-II 9th system, and this SG-II UP facility was commissioned in 2015. More recently, based on a similar technology as the SG-II UP nanosecond system, a new high-power laser prototype of the ICF driver was activated in pursuit of further laser technical improvement, succeeding in exploratory research ${ }^{[18]}$. This prototype completed more than 100 shot assessments at $1 \omega(1.053 \mu \mathrm{m})$ in 2016, and a good performance was obtained. In addition, the SG-II 5 PW facility ${ }^{[19]}$, as an extreme light source, is under construction and the prior two optical parametric amplifiers have been accomplished. All of the present running facilities constitute an open multifunctional platform ${ }^{[20]}$, and many important achievements ${ }^{[21-28]}$, especially for target physics in ICF research, were witnessed in a series of experiments on this platform.

In this paper, the multifunctional experimental platform of the SG-II series facilities is described in Section 2. As the SG-II facility and SG-II 9th laser system have operated for many years, they have already been described in the literature ${ }^{[7,9-15]}$. The newly built SG-II UP facility is described in detail in Section 3; this facility combines many advanced techniques with a good output performance. In addition, the recent developments of the SG-II 5 PW facility have the potential of providing extreme physical conditions, and are introduced briefly in Section 4. Finally, a summary is presented in Section 5.

\section{Status of the multifunctional platform}

The overall layout of the multifunctional platform is shown in Figure 2, showing the three target chambers corresponding to the different facilities. These facilities can be operated independently or combined to support extensive experimental capabilities. Accordingly, this platform is of great significance to interested scientists worldwide, providing various physical experiments, such as fast ignition ${ }^{[25,26]}$ (eight beams used for pre-compression and a picosecond PW beam used for ignition), indirectdrive physics ${ }^{[29-32]}$, direct-drive physics $^{[21]}$, laboratory astrophysics (collisionless shocks) ${ }^{[23,28,33-36]}$, equation of state $^{[24,37]}$, HED physics ${ }^{[27,38]}$, and diagnostic instruments development ${ }^{[24,39-41]}$. Each facility is equipped with a basic set of diagnostics, including a spectrometer (X-ray, visible, and UV), streaked slit camera (X-ray and visible), near backscatter imager, energy disperse spectroscopy (electron/ion/proton), nuclear track analysis system, and neutron angular distribution detector. The general output capabilities and facility features are shown in Table 1.

The SG-II facility with eight beams can deliver $6 \mathrm{~kJ} /$ $1 \mathrm{~ns} / 1 \omega$ and $2.4 \mathrm{~kJ} / 1 \mathrm{~ns} / 3 \omega$ in routine operation. This facility was first used to explore a switch-free, double-pass, and co-axial $2 \times 2$ segment array main amplifier architecture with a beam diameter of $20 \mathrm{~cm}$. Through the precision SG-II program ${ }^{[10]}$, the output average power imbalance of the eight beams achieved a 6\% RMS (root mean square), the pointing accuracy on a cavity target wall surface was measured within a range of $20 \mu \mathrm{m}$ (RMS), and the ability 


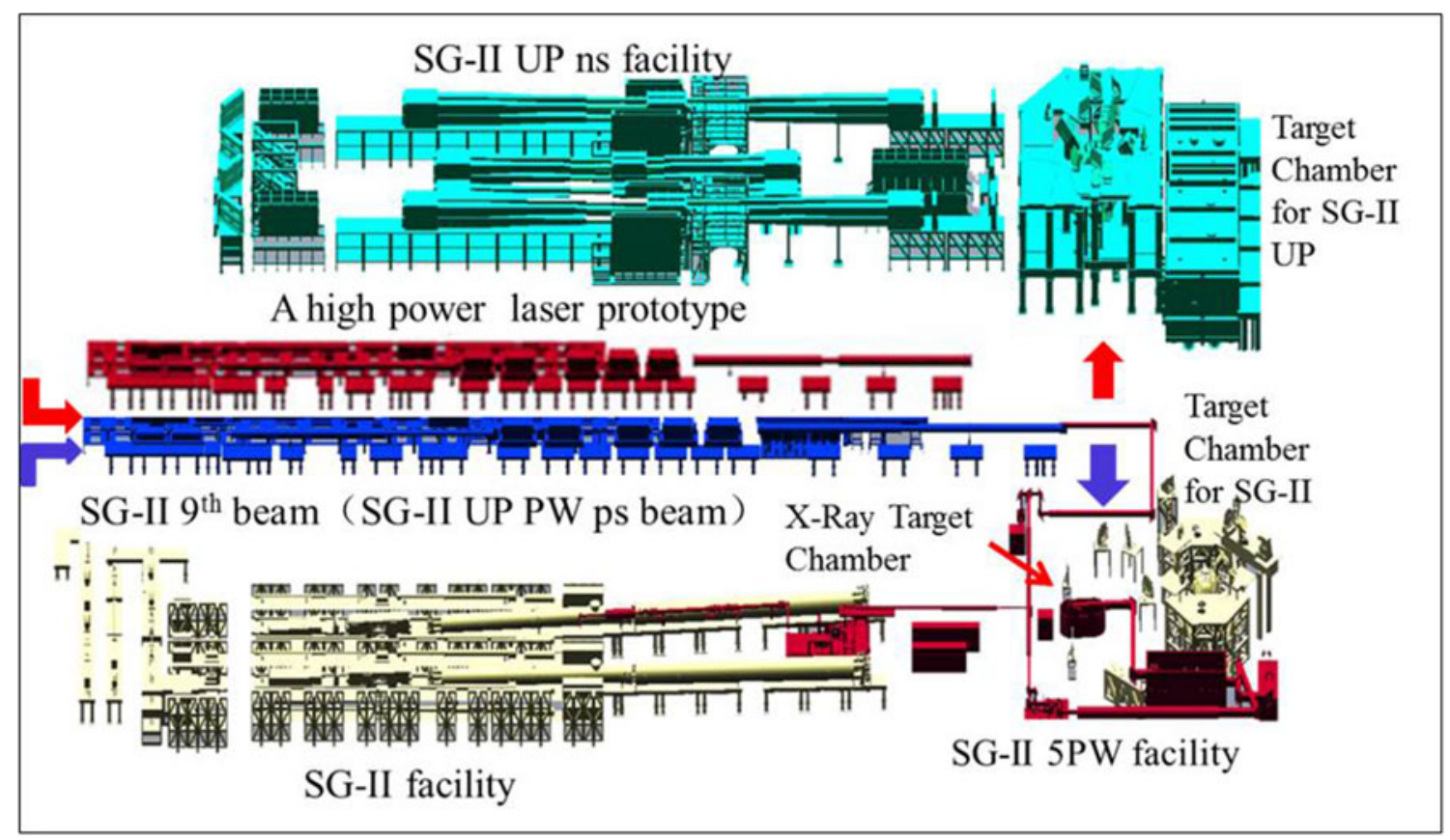

Figure 2. Schematic view of the layout of the multifunctional platform.

Table 1. Output capability and feature of the present facilities.

\begin{tabular}{|c|c|c|}
\hline Facility & Output capability & Feature \\
\hline SG-II facility & $\begin{array}{l}8 \text { nanosecond beams: } \\
0.75 \mathrm{~kJ} / 1 \mathrm{~ns} / 1 \omega / \Phi 20 \mathrm{~cm} / \text { per beam } \\
0.3 \mathrm{~kJ} / 1 \mathrm{~ns} / 3 \omega / \Phi 20 \mathrm{~cm} / \text { per beam }\end{array}$ & $\begin{array}{l}\text { Double-pass, co-axial } 2 \times 2 \text { array main amplifier; good } \\
\text { energy stability; high laser pointing accuracy }{ }^{[10]} \text { and excellent } \\
\text { operation }\end{array}$ \\
\hline SG-II 9th laser system & $\begin{array}{l}5.13 \mathrm{~kJ} / 3.4 \mathrm{~ns} / 1 \omega / \Phi 32 \mathrm{~cm} \\
3 \mathrm{~kJ} / 3.4 \mathrm{~ns} / 3 \omega / \Phi 32 \mathrm{~cm} \\
30 \mathrm{ps}-80 \mathrm{ps} \\
120 \mathrm{ps}-5 \mathrm{~ns}\end{array}$ & MOPA configuration; higher energy; valuable probe beam \\
\hline \multirow[t]{2}{*}{ SG-II UP facility } & $\begin{array}{l}8 \text { nanosecond beams: } \\
5 \mathrm{~kJ}(\max 8.5 \mathrm{~kJ}) / 5 \mathrm{~ns} / 1 \omega / 31 \mathrm{~cm} \times 31 \mathrm{~cm} / \text { per beam (routine } \\
\text { on target) } \\
3 \mathrm{~kJ} / 3.3 \mathrm{~ns} / 3 \omega / 31 \mathrm{~cm} \times 31 \mathrm{~cm} / \text { per beam (routine on target) } \\
\text { laser prototype: } \\
16 \mathrm{~kJ} / 5 \mathrm{~ns} / 1 \omega / 31 \mathrm{~cm} \times 31 \mathrm{~cm} \\
17.5 \mathrm{~kJ} / 20 \mathrm{~ns} / 1 \omega / 31 \mathrm{~cm} \times 31 \mathrm{~cm}\end{array}$ & $\begin{array}{l}\text { Large-aperture four-pass } 2 \times 2 \text { main amplifier; good beam } \\
\text { quality; flexible pulse shaping; higher fluence }\end{array}$ \\
\hline & $\begin{array}{l}\text { PW picosecond beam: } \\
1 \mathrm{~kJ} / 1-10 \mathrm{ps} / 1 \omega / \Phi 32 \mathrm{~cm} \\
\left.10^{20} \mathrm{~W} / \mathrm{cm}^{2}, \mathrm{SNR}>10^{-8} \text { (before }-81.75 \mathrm{ps}\right)\end{array}$ & $\begin{array}{l}\text { Large energy; high focal power density; high signal to noise } \\
\text { ratio; OPCPA + CPA amplification; fast ignition research }\end{array}$ \\
\hline SG-II 5 PW system & $\begin{array}{l}5 \mathrm{PW} / 808 \mathrm{~nm} / 29 \mathrm{~cm} \times 29 \mathrm{~cm} \text { (designed) } \\
1.76 \mathrm{PW} / 808 \mathrm{~nm} / 21 \mathrm{fs} / 29 \mathrm{~cm} \times 29 \mathrm{~cm} \\
\text { (phase two) }\end{array}$ & $\begin{array}{l}\text { Ultra-short higher power; OPCPA amplification new function } \\
\text { and technology }\end{array}$ \\
\hline
\end{tabular}

of flexible pulse shaping was implemented. This facility, greatly promoting Chinese ICF research, has had a stable and excellent operation for approximately 20 years; it will be mainly employed for several expansion techniques in the future.

The SG-II 9th system, a master oscillator power amplifier (MOPA) system, can provide $3 \mathrm{~kJ} / 3 \mathrm{~ns} / 3 \omega$ output in normal operation with a large amplifier aperture of $35 \mathrm{~cm}$. This system has the significant ability of adjusting the pulse width in a wide range from tens of picoseconds to nanoseconds, and can generate different frequency outputs of $1 \omega, 2 \omega, 3 \omega$, and $4 \omega$ with adjustable polarization. When coupled with the SG-II facility, it acts as an exceptional laser probe with a high synchronization precision of $<4.3 \mathrm{ps}$ (RMS). Therefore, many worthy experimental results have been produced by these two facilities and the total operational shots over the past 17 years are given in Figure 3. By December 31st, 2017, a total of 7433 shots had been produced by the SG-II facility for physical experiments. No physical experiments were carried out in 2007 because of a laboratory transformation.

The SG-II UP facility is composed of two parts; the SGII UP nanosecond facility and the SG-II UP PW picosecond 


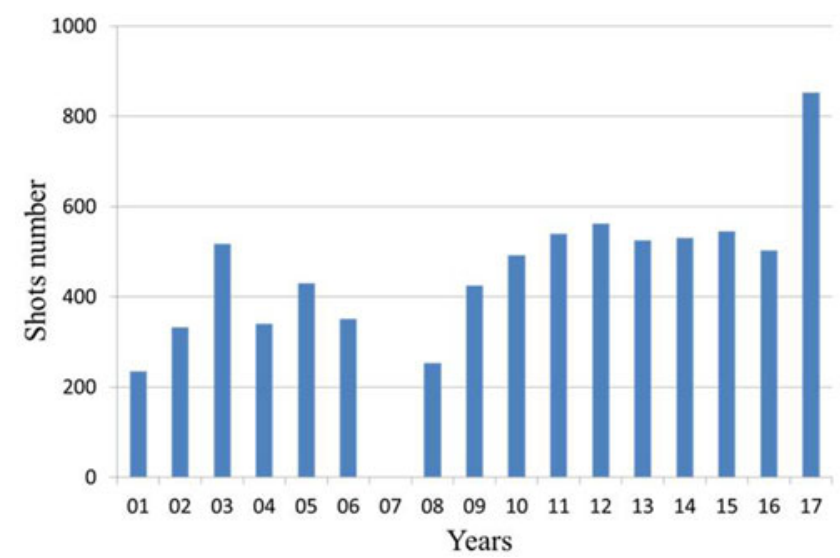

Figure 3. Operational shots of SG-II facility for physical experiments.

system. The SG-II UP nanosecond facility with eight beams is designed to provide $24 \mathrm{~kJ} / 3 \mathrm{~ns} / 3 \omega$ output with the fullbeam size of $31 \mathrm{~cm} \times 31 \mathrm{~cm}$ (beam size at the level of $1 \%$ of the maximum fluence). However, the actual output is greater than this. The facility adopts large-aperture four-pass cavity amplification and two-pass power amplification installed in a $2 \times 2$ arrangement. In order to verify the larger output capability at $1 \omega$, we have developed a high-power laser prototype. This prototype with eight beamlines is similar to the SG-II UP nanosecond facility, whereas the maximum $1 \omega$ output of this prototype is greater; a $>100$ shots assessment validated that the prototype is capable of delivering $16 \mathrm{~kJ} / 5 \mathrm{~ns} / 1 \omega$ and $17.5 \mathrm{~kJ} / 20 \mathrm{~ns} / 1 \omega$. This prototype is of great importance to study the $3 \omega$ damage problem in depth and other technology developments. Furthermore, it has the potential of providing an output of $10 \mathrm{~kJ} / 3 \omega$. In addition, the SG-II UP petawatt picosecond system based on the SG-II 9th amplification chain can supply $1 \mathrm{~kJ} / 1-10$ ps to the SG-II UP target chamber; this petawatt picosecond system utilizes the chirped-pulse amplification (CPA) technique, providing the maximum energy and pulse compression technique to obtain a very short pulse width. The maximum power density and signal to noise ratio (SNR) are $10^{20} \mathrm{~W} / \mathrm{cm}^{2}$ and $10^{8}$, respectively. By December 31st 2017, a total of 719 shots had been produced on the SG-II UP facility over a four year period for use in physical experiments (see Figure 4).

The SG-II 5 PW system is designed to provide femtosecond multi-petawatt pulses, employing a three-stage non-collinear optical parametric chirped-pulse amplifier (OPCPA). To date, the previous goals for the two optical parametric amplifiers have been fulfilled, and a chirped pulse with energy of $49.7 \mathrm{~J}$ and full-width-at-half-maximum (FWHM) spectrum bandwidth of $85 \mathrm{~nm}$ has been achieved. After being compressed, the pulse was greater than $37 \mathrm{~J}$ in $21 \mathrm{fs}(1.76 \mathrm{PW})$, and the focal spot was $\sim 10 \mu \mathrm{m}$ after closed-loop correction by the adaptive optics (AO) when all amplifiers were off. At present, the pulse can be

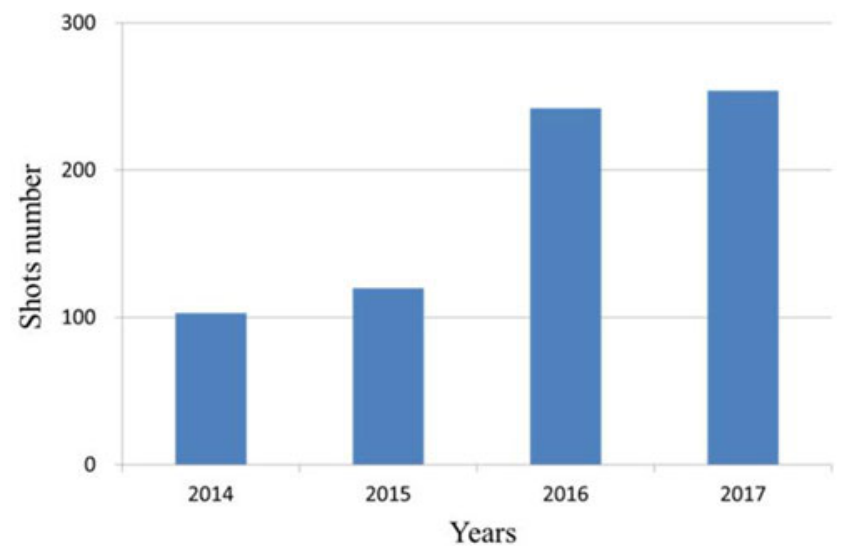

Figure 4. Operational shots of SG-II UP facility for physical experiments.

directed to an X-ray target chamber for high-energy physical experiments and laser proton acceleration has been realized.

\section{Performance of the SG-II UP facility}

The SG-II UP facility is characterized as a typical and new multi-pass amplifier system based on a large-aperture plasma electrode Pockels cell (PEPC) at the NLHPLP. It took nearly 6 years to construct and complete the SG-II UP facility engineering and the demand of the output characteristics for the facility was ultimately satisfied. Many advanced technologies have been adopted to support its construction and some critical optical components have been provided by many suppliers to guarantee the laser performance. Two great developments have been achieved, (1) the transition of the main configuration from a co-axis double-pass of the SG-II facility to the four-pass amplification of the SG-II UP nanosecond system and (2) the realization of a high-energy petawatt laser ${ }^{[42]}$ based on CPA using Nd:glass solid-state amplifiers. Photographs of the SG-II UP facility are shown in Figure 5.

The SG-II UP facility has been in normal operation for physical experiments since 2016. The nanosecond and picosecond systems can be fired independently or flexibly combined. The picosecond laser system can be used to conduct five shots for a physical experiment each day. The SG-II UP facility can support a variety of HED physical experiments, such as fast ignition and fast electron acceleration and proton radiography. More recently, the SG-II UP facility completed the first-phase indirect-drive fast ignition integrated experiment. In this experiment, the observed neutron gain reached a value of $>44$ times, demonstrating that the picosecond laser can effectively heat fuel ${ }^{[25]}$. In addition, in the laser proton accelerated experiment, proton beams $>50 \mathrm{MeV}$ were measured by a Thomson ion spectrometer. Furthermore, experimental 


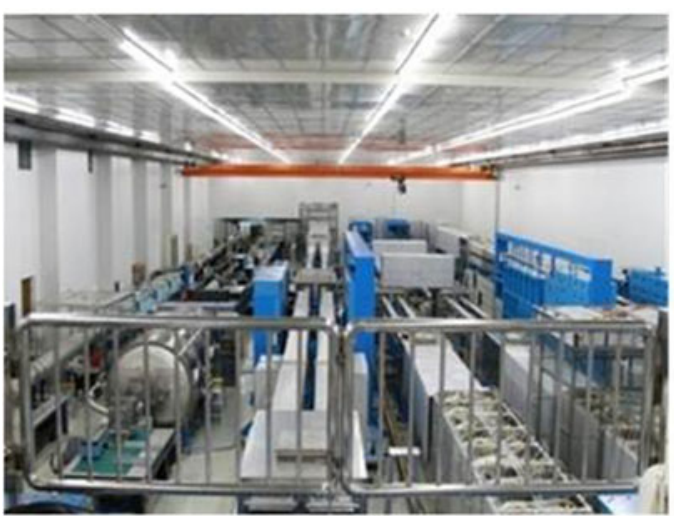

(a)

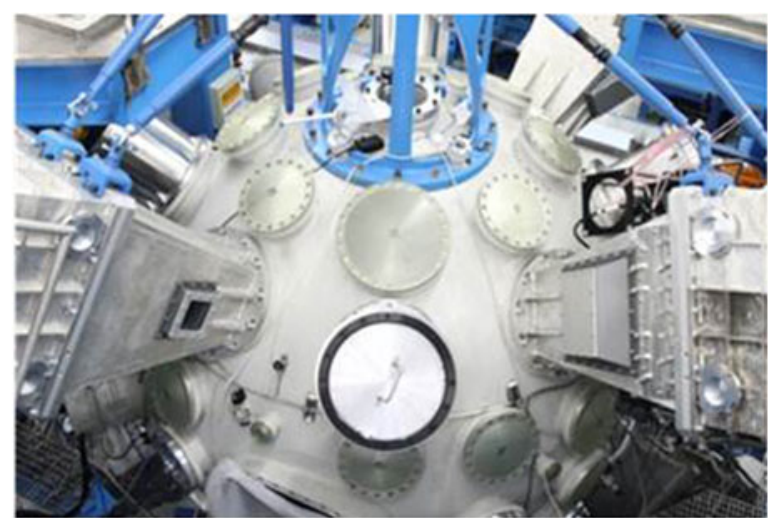

(b)

Figure 5. Photographs of the SG-II UP facility: (a) laser hall and (b) target chamber.

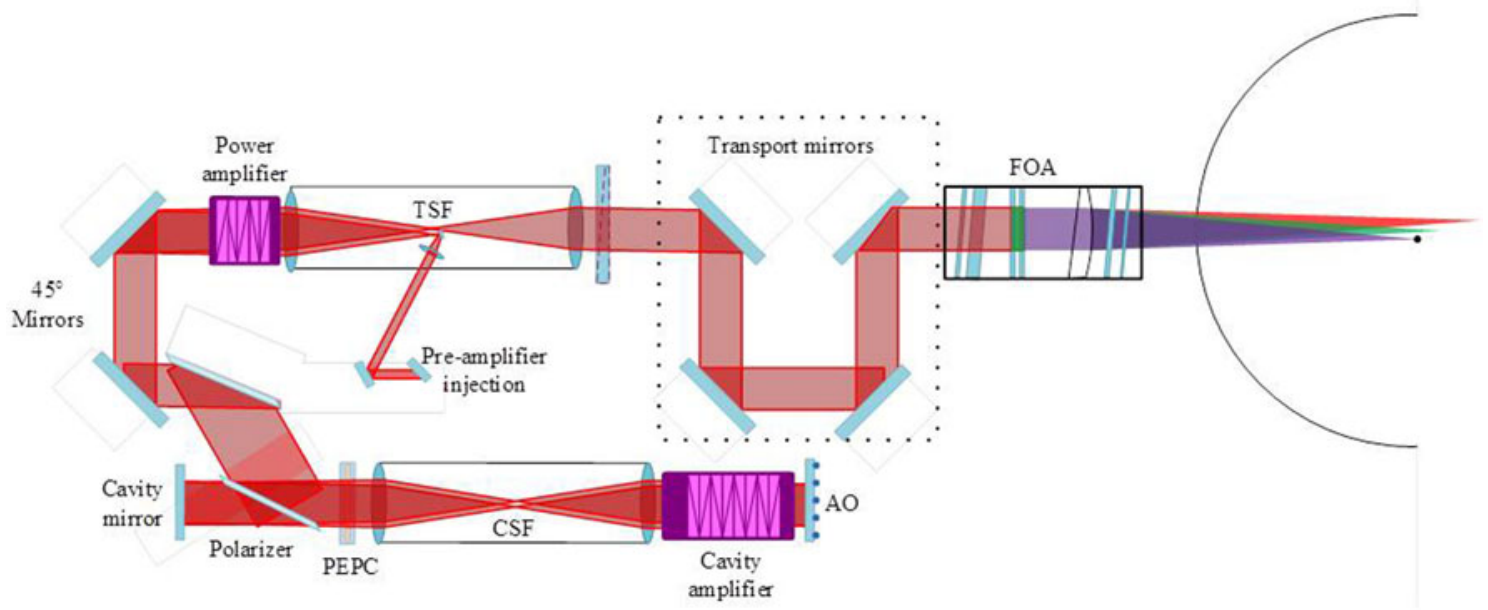

Figure 6. Schematic of the optical layout of one beamline.

evidence of kinetic effects in indirect-drive ICF hohlraums was shown by the proton radiography on SG-II UP laser facility ${ }^{[43]}$.

\subsection{SG-II UP nanosecond system}

\subsubsection{Optical layout}

A schematic diagram of the overall layout of one beamline of the SG-II UP nanosecond system is shown in Figure 6. A laser pulse at a nanojoule level energy with a center frequency at $1053 \mathrm{~nm}$ is first generated in the front-end subsystem, where the pulse shaping and frequency fine tuning are also optimized ${ }^{[44]}$. Then it is successively preamplified to an approximate joule level in the preamplifier module where the spatial control is achieved. Afterward, the pulse is injected into the main amplifier system. As illustrated in Figure 6, there are two bundles containing four beamlines in a $2 \times 2$ array in the main amplifier system and the four beamlines share the common preamplifier. The main amplifier system of one beamline includes two amplifiers distributed in two parallel lines: the cavity amplifier (eight slabs) and power amplifier (five slabs). All of the slabs are mounted at Brewster's angle and are N31 type neodymium doped phosphate glass with thickness $45 \mathrm{~mm}$. The phosphate glass was developed at the Shanghai Institute of Optics and Fine Mechanics ${ }^{[45]}$. The pulse passing through the power amplifier is directed to a cavity amplifier section by three mirrors. A PEPC ${ }^{[46]}$ is utilized in conjunction with a large-aperture polarizer ${ }^{[47]}$ to control the pulse transport out from the cavity section. Adaptive optics is adopted in the cavity amplifier section to correct the pulse wavefront ${ }^{[48,49]}$. Meanwhile, two confocal spatial filters, namely the transport spatial filter (TSF) and cavity spatial filter (CSF), are used to restrain the beam modulation. As the pulse again propagates through the power amplifier, after passing the TSF and transport mirrors, it enters the final optics assembly (FOA) ${ }^{[50]}$ and it is frequency converted to $351 \mathrm{~nm}$ light, before being focused to the target chamber using the wedged focus lens. 


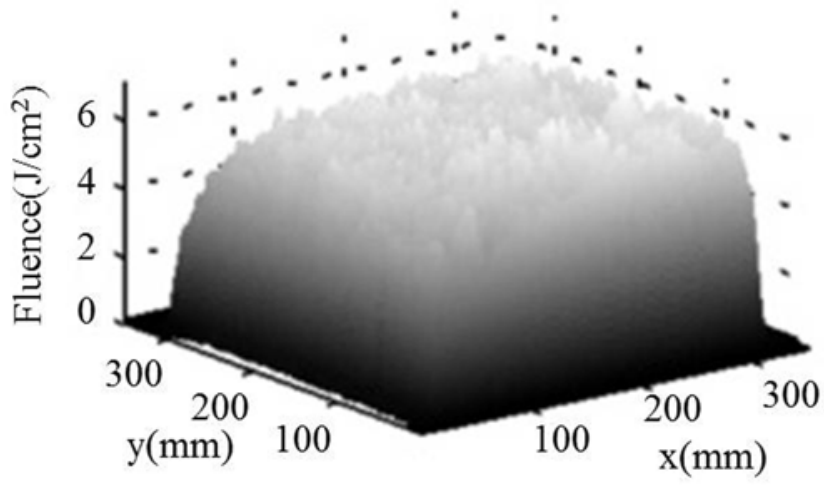

(a)

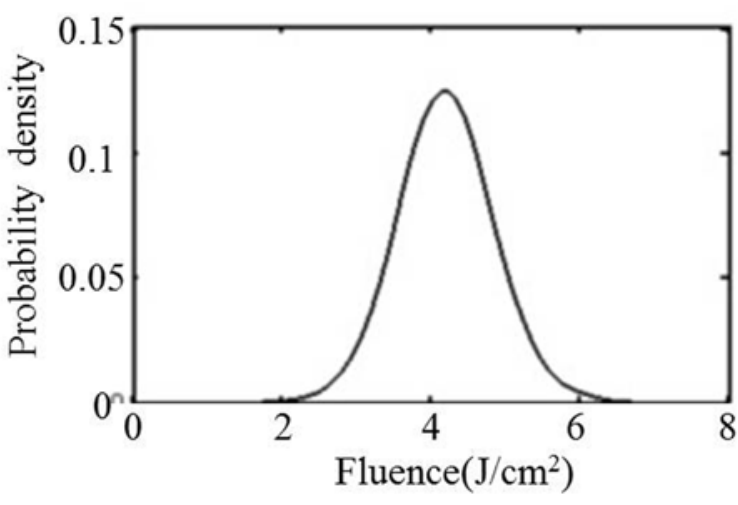

(b)

Figure 7. Near-field fluence distributions of the $1 \omega$ output (shot No. 20150721002): (a) near-field images and (b) fluence probability distribution.

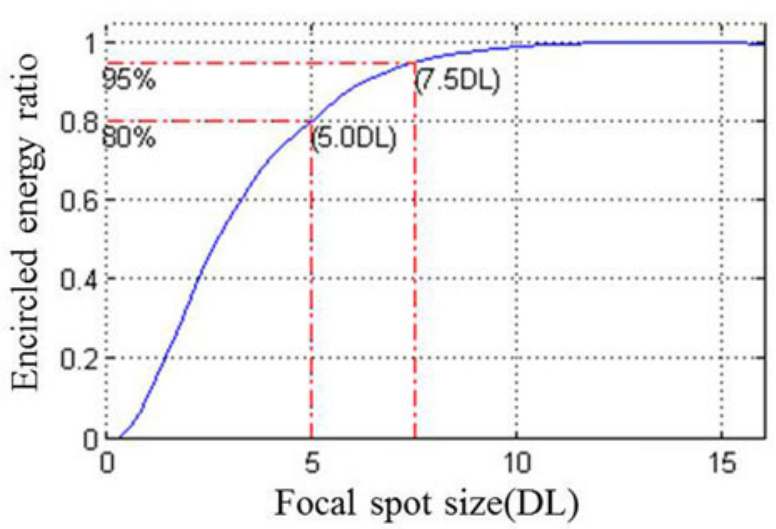

(a)

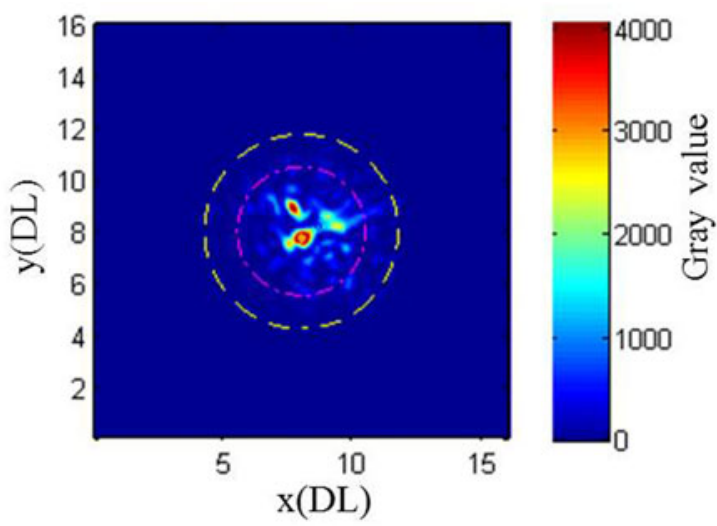

(b)

Figure 8. Far-field fluence distributions of the $1 \omega$ output (shot No. 20150721002): (a) enclosed focal spot energy fraction and (b) far-field image.

In addition, the measurement system ${ }^{[51-53]}$, centralized control system ${ }^{[54,55]}$, and automatic alignment system ${ }^{[56,57]}$ are all involved to enable the effective operation of the facility.

\subsubsection{Output ability}

The SG-II UP nanosecond system with all eight beamlines can be operated at a $\geqslant 5 \mathrm{~kJ} /$ beamline for a $1053 \mathrm{~nm}$ wavelength demonstrating a $1 \omega$ capability of $>40 \mathrm{~kJ}$. The nearfield characteristic of one beam with a $1 \omega$ output energy of $\sim 5 \mathrm{~kJ}$ is shown in Figure 7. It is demonstrated that the near field has good uniformity with a fluence beam contrast ratio (FBC) 0.08. FBC is defined as the standard deviation in the fluence profile divided by its average value ${ }^{[4]}$. The far field of the same shot is shown in Figure 8, with a good quality of the far field as well; the $95 \%$ and $80 \%$ focused energies are encircled inside 7.5 DL (diffraction limit) and 5 DL, respectively. It should be noted that due to some aspects, such as project needs, engineering costs, and construction foundations, the main amplifier systems for the eight beamlines did not employ the best configuration. This later inspired the actual technical improvements of the SG-II UP nanosecond facility; a high-power laser prototype was launched to further pursue the optimization of the laser design and additionally test the $1 \omega$ output potential with investigations of the final optical issues. With some parameters changed by optimization and technical upgrade ${ }^{[55-64]}$, the outputs of $16 \mathrm{~kJ} / 5 \mathrm{~ns} / 1 \omega$ and $17.5 \mathrm{~kJ} / 20 \mathrm{~ns} / 1 \omega$ were achieved based on $16 \mathrm{Nd}$ :glass slabs (11 slabs in the cavity amplifier and 5 slabs in the power amplifier) with $40 \mathrm{~mm}$ thickness. More than 100 shots with different pulse widths and energies in the $1 \omega$ output were finished in 2016. Figure 9 provides a summary of the $1 \omega$ shots fired together with an operating constraint envelope. This constraint (dashed line in Figure 9) is set by considering several aspects, such as the nonlinear growth, beam quality trend, and degree of optic damage. An excellent performance of this prototype was verified and the higher energy output also implies an extremely high fluence density of $>19 \mathrm{~J} / \mathrm{cm}^{2}$, compared with amplifiers of apertures $>40 \mathrm{~cm}$. The output ability is appreciable and adequate to support the $3 \omega$ output. Moreover, the $1 \omega$ beam could be expanded to facilitate the final output with a larger aperture for $3 \omega$ optical components. For a detailed description of the performance 


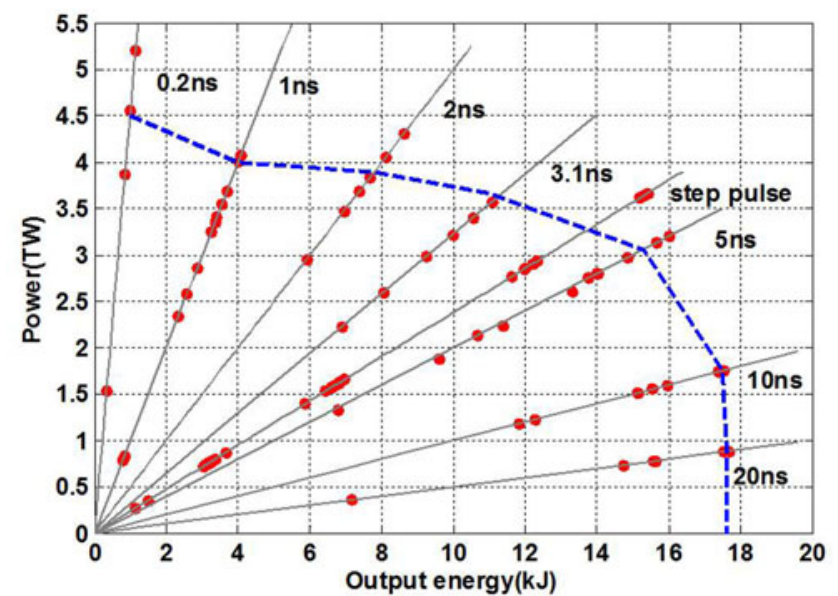

Figure 9. Experimental output capability for $1 \omega$ with different pulse widths of the laser prototype.

of this prototype, see Ref. [18]. It should be noted that the measured small signal gain coefficient of the main amplifier was $\sim 5 \% \mathrm{~cm}^{-1}$. When the self-oscillation within the main system was determined and measures were taken to suppress the self-oscillation, the small signal gain coefficient of the main amplifier reached $5.2 \% \mathrm{~cm}^{-1}$, with further testing required to determine the potential.

The $3 \omega$ experimental output capability ${ }^{[65]}$ with different pulse widths for the SG-II UP nanosecond system and laser prototype is shown in Figure 10; eight beamlines were delivered to the target chamber and frequency converted to $3 \omega$ light. The output energy of a single beam is $3 \mathrm{~kJ} / 3.3 \mathrm{~ns}$. The maximum energy is $5 \mathrm{~kJ} / 4.6 \mathrm{~ns}$ and corresponding maximum fluence is $5.2 \mathrm{~J} / \mathrm{cm}^{2}$. The total output energy of eight beams is $25 \mathrm{~kJ} / 3.3 \mathrm{~ns}$ in routine operation. We have developed a precision diagnostic system (PDS) based on a type of off-axis imaging system with three spherical mirrors ${ }^{[52]}$ to measure the key parameters, such as the near and far fields. The near and far fields with a $3 \omega$ frequency and $1 \omega$ energy of $8.5 \mathrm{~kJ}$ are shown in Figure 11 . We obtained a relatively good near-field distribution and the fluence beam contrast of the near field was 0.17 with a $3 \omega$ energy output of $3.3 \mathrm{~kJ} ; 95 \%$ of the focused energy was approximately encircled inside $18 \mathrm{DL}$. In addition, from Table 1 there is a gap between the $1 \omega$ and $3 \omega$ output abilities in routine operation. Due to the high fluence output of $1 \omega$, and current $3 \omega$ operation limit owing to optics damage, the beam expanding method can be used to decrease the $3 \omega$ fluence and effectively utilize the $1 \omega$ output. Importantly the beam quality may be improved through beam expanding and clearly more effort should be made to improve the damage resistance at $3 \omega$.

The laser power balance is one of the primary criteria representing the overall performance of a laser facility. The laser energy stability is a key factor for the final power imbalance, so the laser energy fluctuation must be

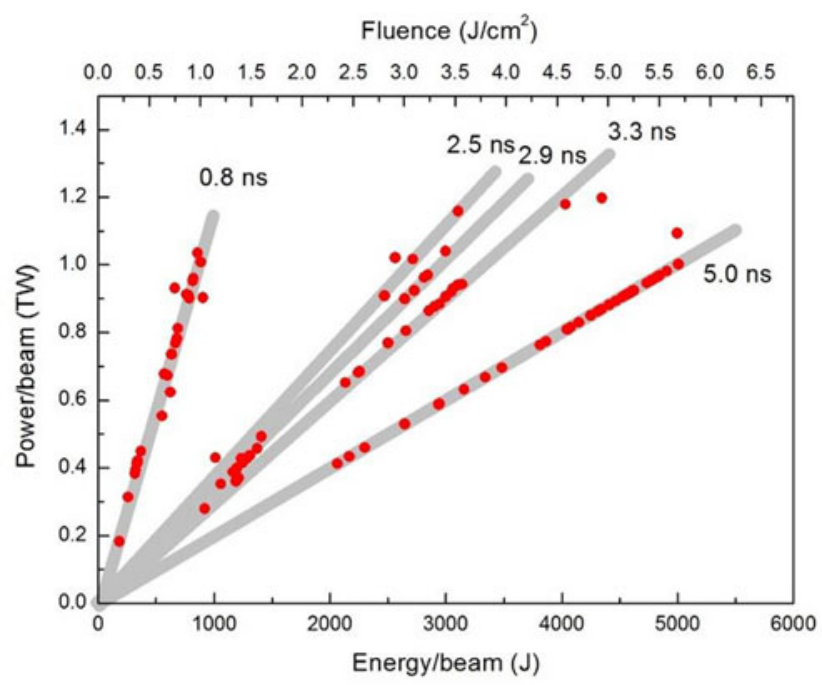

Figure 10. Experimental output capability of $3 \omega$ with different pulse widths.

carefully controlled during the whole laser propagation and amplification. The energy fluctuation of all eight beams of the SG-II UP nanosecond facility for four consecutive shots is shown in Figure 12, where the average energy fluctuation of all eight beams is $0.9 \%$. The beam-to-beam energy imbalance is $1.9 \%$ (RMS). The beam-to-beam instantaneous power imbalance is characterized as an RMS value of the instantaneous power fluctuation between different beams on the same shot. The beam-to-beam RMS instantaneous power is then averaged over a specified time interval to calculate the average RMS power imbalance. For the square wave, the time interval between the rise and fall time point at half the peak intensity is used to obtain the average RMS power imbalance. The results of the power balance from experimental shot 4 are displayed in Figure 13. The average $3 \omega$ energy of the eight beams was $3114.5 \mathrm{~J}$ and the average power imbalance between the eight beams for the 3-ns square wave was $4.7 \%$ (RMS).

A flexible pulse shaping stability is important for power balance control and at present different pulse shapes can be generated with high accuracy, mainly, the Haan pulse ${ }^{[4]}$ and step pulse (or named four-shock-shaped pulse) ${ }^{[66]}$. This capability is possible as a result of pulse shaping technology combined with an automatic close-loop control technique. Figure 14 shows the experimental pulse shape generated in the front end and output pulse shape at the end of the main amplifier on the laser prototype. It is demonstrated that the experimental Hann pulse output (solid line) is close to the expected pulse shape (dashed line). With a 2-ns window error analysis, the time of the waveform deviation between the actual and expected outputs in the whole window is within $10 \%$, and the deviation of the main pulse is $<3 \%$. Overall, the high energy and power balance assures a high stability and high success rate for physical experiments using the SG-II UP nanosecond facility. 


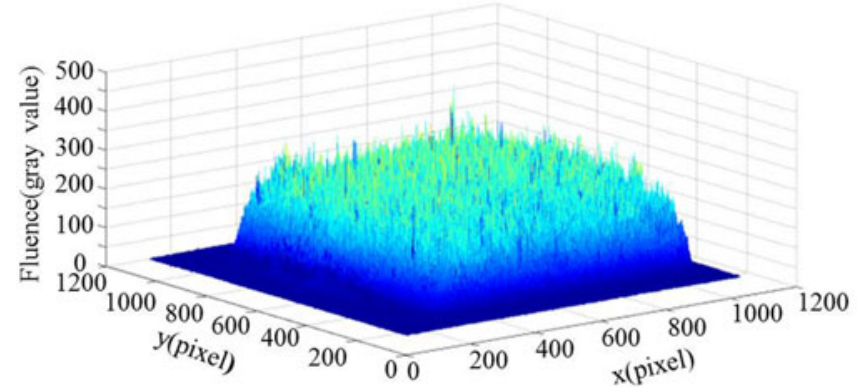

(a)

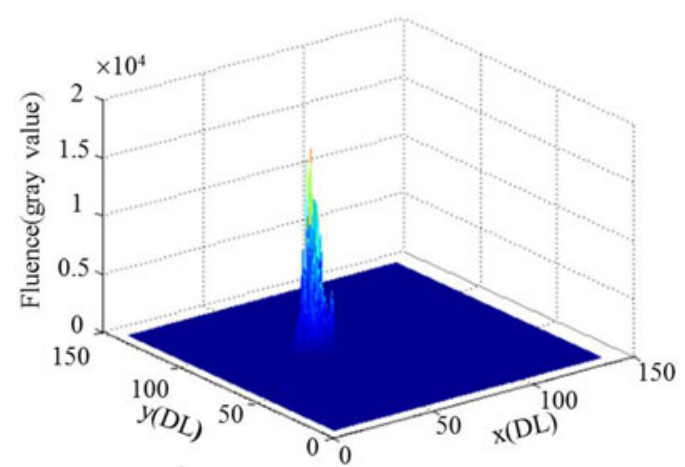

(b)

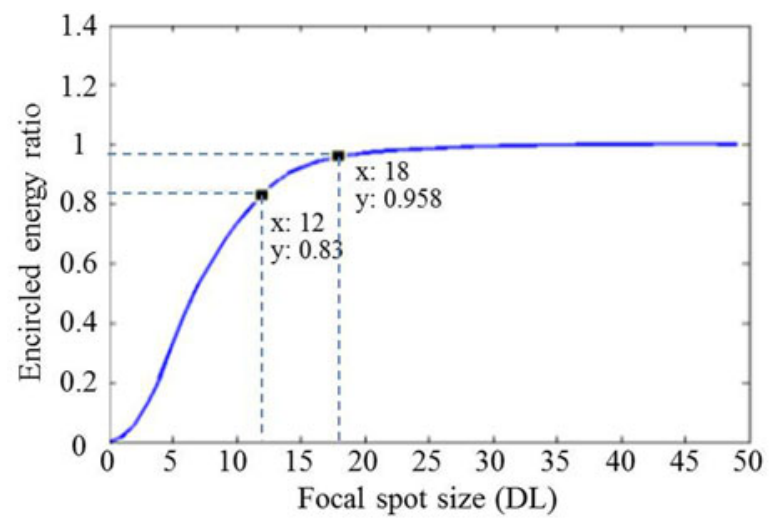

(c)

Figure 11. Near and far fields of the $3 \omega$ output measured by PDS: (a) near-field image, (b) far-field image, and (c) enclosed $3 \omega$ focal spot energy fraction.

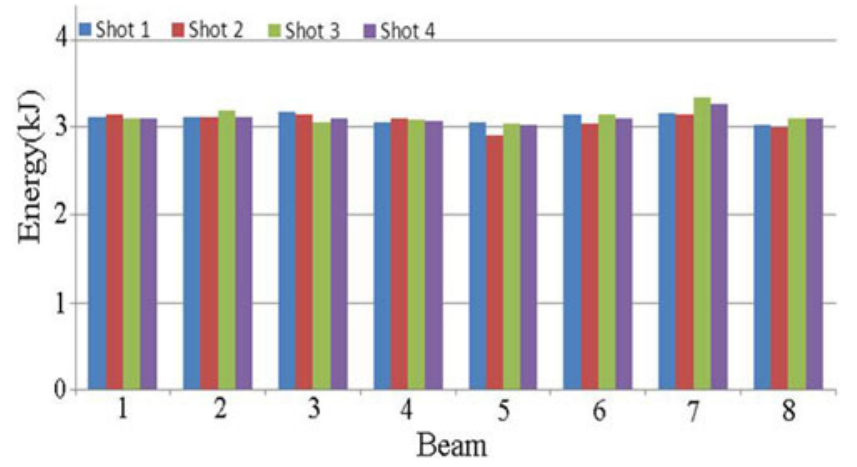

Figure 12. $3 \omega$ output energy per beam for four consecutive shots.

It is known that the precise and stable synchronization between different beams is necessary for positive diagnostics, fast ignition research, and high performance implosion research. The SG-II UP facility utilizes the fiber stacker, high-speed electronics, and analog to digital conversion technology ${ }^{[44]}$ to realize a simultaneous long and short pulse timing precision of $<4.1 \mathrm{ps}$ (RMS) within a 2-h test. In addition, high-precision synchronous trigger signals ${ }^{[67]}$, optical and electrical signals for multi-channel physical tests, and diagnostics have been established. The time jitter between the signal and main laser reaches $\leqslant 3$ ps (RMS) and 20 ps (PV).

\subsubsection{Main technical developments}

Overall, the SG-II UP nanosecond system has driven the fabrication and processing of large optical components ${ }^{[68]}$; furthermore, during the construction it provided many technical developments that ensured a good performance of the system. We introduce some of these as follows.

(1) The introduction to the overall design of a four-pass amplifier configuration based on a large-aperture PEPC is demonstrated. We have written a set of simulation software called Laser Designer ${ }^{[69]}$. In terms of the overall design, the requirements of the output energy, beam quality, safe operation (low risk of optical damage), and physical experiments of the facility were fully considered. Redundant design for the output capacity was adopted to meet the output energy requirement and extend the service life of the facility for physical experiments. In fact, we designed the normal operating energy of the facility based on a cumulative B integral equal to 2.8 , equivalent to $5 \mathrm{~kJ} / 3 \mathrm{~ns} / 1 \omega$.

For the beam quality, a design incorporating whole system image transfer and aberration elimination was adopted. For example, in the preamplifier system, a zoom spatial filter was used to compensate the optical path difference of the whole system, and the image relaying of eight laser beams was consistent. In the same way, the geometric aberration of the preamplifier system was compensated by the combined 


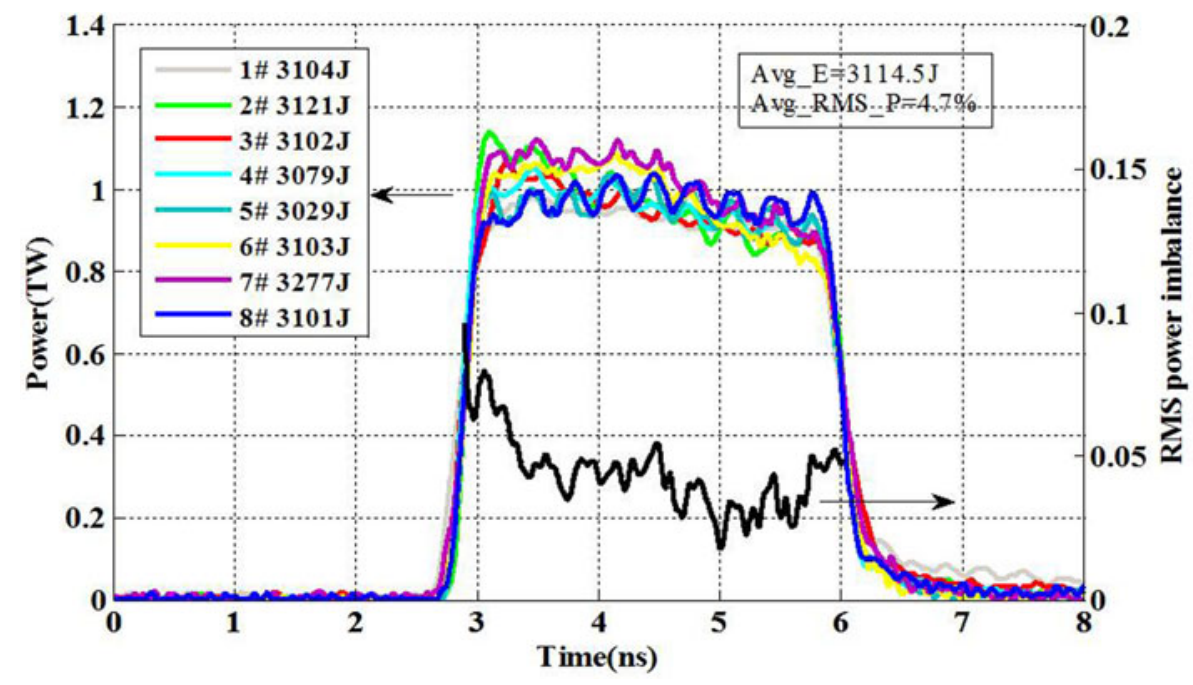

Figure 13. $3 \omega$ output power imbalance for eight beams (shot 4 ).
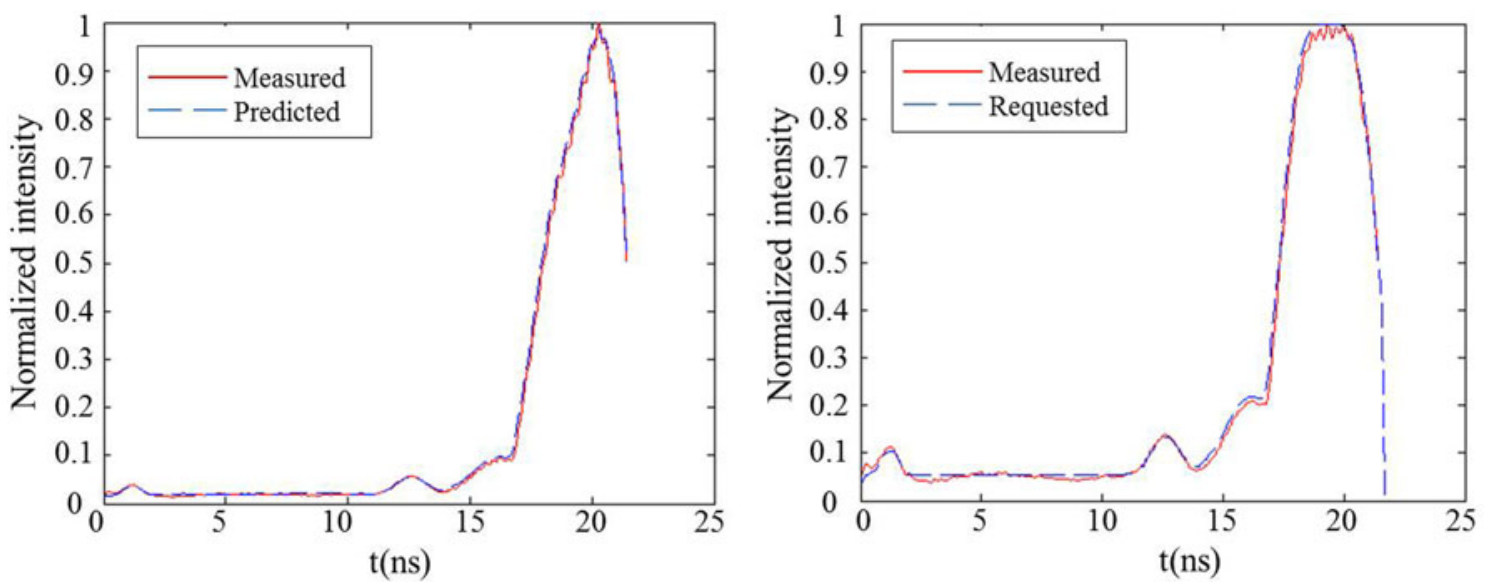

Figure 14. Pulse shape in the (a) front end and (b) end of the main amplifier.

lens, and the geometric aberration of the main amplifier system was compensated by the non-spherical lens. Therefore, reasonable elimination of aberration for the whole system was achieved.

To minimize the risk of optical damage the design avoided ghost images of the first to third order in the main amplifier system, and ghost images of the first to fourth order in the final optics system. All types of stray light in the optical path were simultaneously blocked and appropriately absorbed. In order to obtain high contrast and square wave pulses required for physical experiments, the characteristics of the laser pulse temporal waveform shaping were analyzed in detail through the constant correction and improvement of the parameters in the software based on a series of laser shots. The simulation results agreed well with the experimental results, as shown in Figure 15.

(2) Flexible control techniques in the front-end, preamplifier system, and a high stability regenerative amplifier were developed. We independently researched and developed a polarization auto-feedback adjustment module based on fiber optic squeezing ${ }^{[44]}$. This technique improved the output stability of the nanosecond system. A regenerative amplifier has many advantages, including high gain, high stability, wide spectrum amplification, and high output beam quality, and therefore a high stability wide spectrum regenerative amplifier ${ }^{[70,71]}$ was independently developed, a photograph of which is shown in Figure 16(a). This regenerative amplifier with a good near-field quality [Figure 16(b)] was operated with a $1 \mathrm{~Hz}$ repetition rate. With an injected pulse energy of $500 \mathrm{pJ}$ the output energy reached a maximum of $10 \mathrm{~mJ}(5 \mathrm{~ns})$ after the pulse passed 33 round trips in the cavity. The stability of the output energy for more than $8 \mathrm{~h}$ was better than $0.3 \%$ (RMS) as shown in Figure 16(c), and it is demonstrated in Figure 16(d) that the square-pulse distortion (SPD) defined as the ratio of the gain at the leading edge of a square pulse to that at trailing edge of the pulse $\mathrm{s}^{[72]}$ was less than $1.3: 1$. 


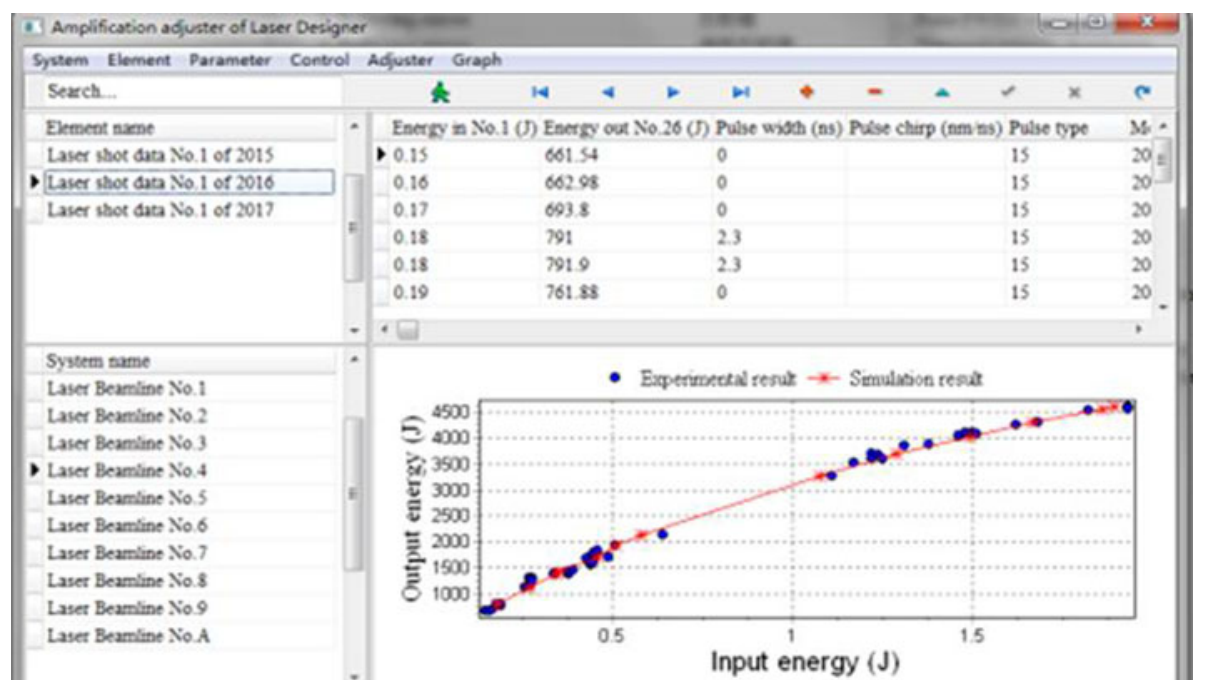

Figure 15. Interface of Laser Designer and the comparison of the experimental and simulation results.

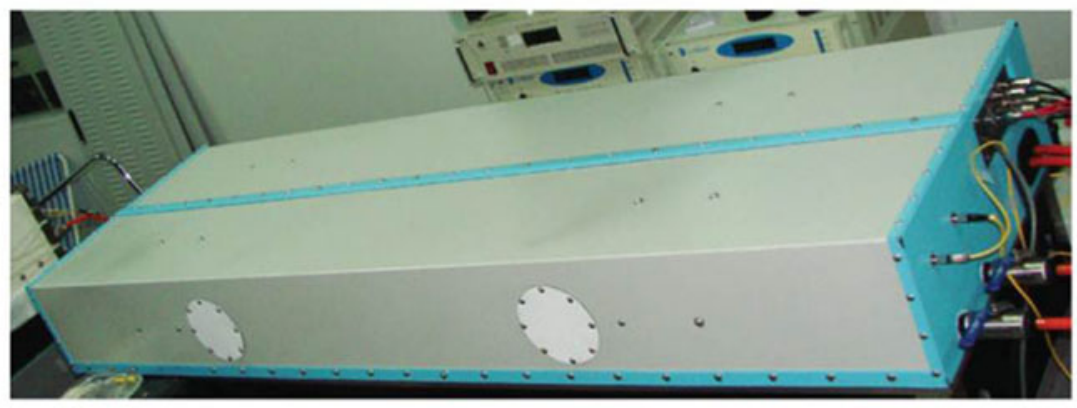

(a)

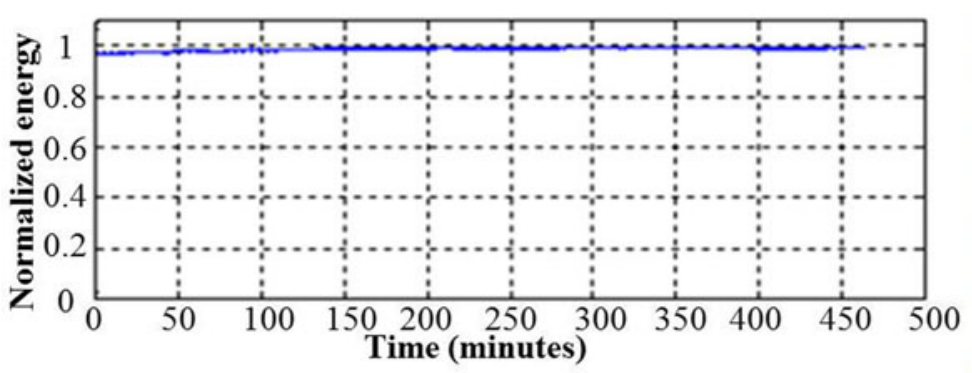

(c)

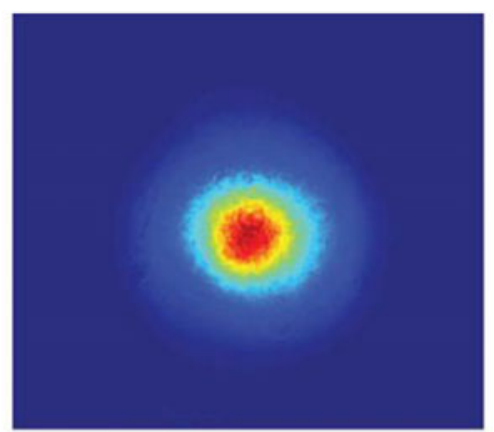

(b)

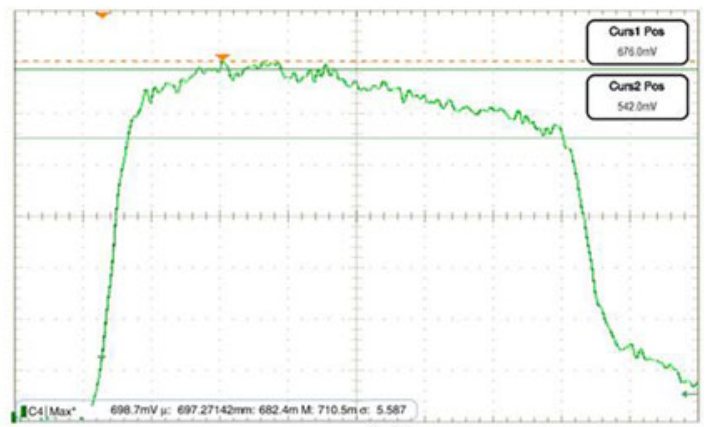

(d)

Figure 16. (a) Regenerative amplifier, (b) output near-field profile, (c) energy stability of the regenerative amplifier for 8 h, and (d) square-pulse distortion of the regenerative amplifier.

The spatial shaping in the preamplifier system is important for a good near-field distribution of the final output and improved operating fluence. We adopted spatial beam shaping step by step. A binary mask was used in the front of the preamplifier section to form the soft-edge and beam profile from round to square shape, and another binary mask was used at the end of the preamplifier section to further sharpen the intensity soft edge for improving the energy extraction of main amplifier. An optically addressed spatial light modulator (SLM) developed in $2012^{[73]}$, was utilized to pre-compensate for the nonuniformity of the spatial gain distribution and to pre-block the damage sites of the largeaperture optics. Through a series of optimization, this efficient and integrated optically addressed SLM achieved good performance characteristics. A transmission of up to $85 \%$ was obtained (for $1053 \mathrm{~nm}$ ), wavefront deviation was $<0.5 \lambda$ 


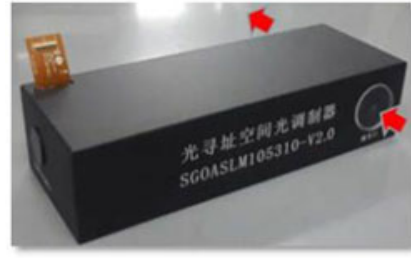

(a)

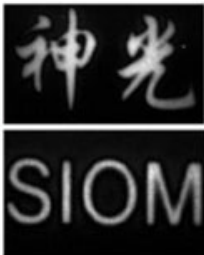

(b)

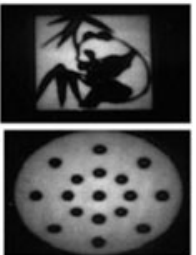

Figure 17. (a) Physical photograph of the optically addressed liquid crystal spatial light modulator and (b) demonstration of near-field spatial intensity control.

(for $\Phi 10 \mathrm{~mm}$ ), damage threshold reached $100 \mathrm{~mJ} / \mathrm{cm}^{2}$, effective application area was $22 \mathrm{~mm} \times 22 \mathrm{~mm}$, and on-off ratio was $100: 1$. A photograph of the optically addressed SLM is shown in Figure 17(a). A demonstration of the spatial control provided by this device is shown in Figure 17(b).

(3) An alignment system has been designed and applied that takes advantage of the imaging characteristics of a multi-pass amplifier. The alignment system ${ }^{[56,74,75]}$ in the SG-II UP nanosecond facility mainly contains the automatic alignment of the preamplifier, main amplifier, and harmonic conversion systems. According to the features and requirements of the facility, the alignment process was appropriately designed, and alignment sensor packages were developed. Many operational results have shown that all of the functionalities have been implemented, including the farand near-field alignments. The alignment accuracy of the far field was better than $2.8 \mu \mathrm{rad}$ in TSF-2 pinhole and $2.2 \%$ in TSF-1 pinhole and all CSF pinholes, and the alignment accuracy of the near field was better than $0.32 \%$ of the local beam aperture. In addition, the conversion crystal alignment accuracy was improved to better than $4.7 \mu \mathrm{rad}$. All the results indicate that the alignment system of the SG-II UP nanosecond laser facility is highly accurate, reliable, and easy to use. Alignment process was completed within $30 \mathrm{~min}$ before every shot during the routine operation of the facility.

The alignment system for the main amplifier and harmonic conversion crystals of FOA is defined here. The main amplifier of the SG-II UP nanosecond laser facility is shown in Figure 18. The far-field alignment sensors were located in the TSF and CSF and the mirrors used for automatic alignment are indicated in Figure 18.

The CSF alignment package was utilized to align the far field of the four-pass laser beams, as shown in Figure 19. The package was composed of an illumination LED (lightemitting diode), a set of imaging lenses, a grating, a halfwave plate, and a detector (CCD). Four sets of far-field references corresponding to four passed beams (four symmetric circular regions in each set) were fabricated on the grating. As the laser beam and LED illumination beam irradiated the transmission grating, their +1 order diffraction beams were collected by the imaging components and an image indicating the information about the relative position of focus point and far-field reference was obtained. Here LED positioned at -1 order diffraction direction was used
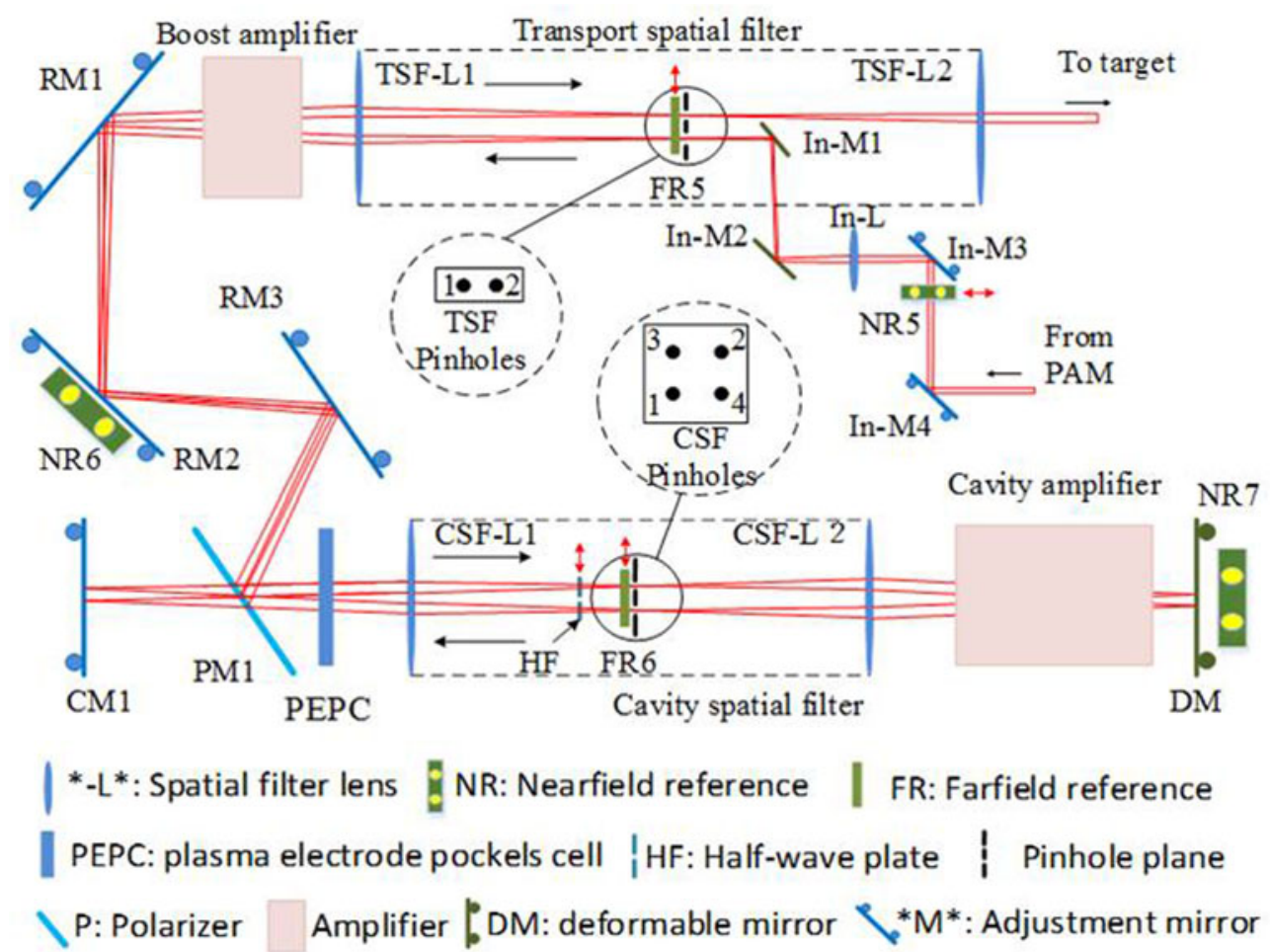

Figure 18. Main amplifier of the SG-II UP ns laser facility. 


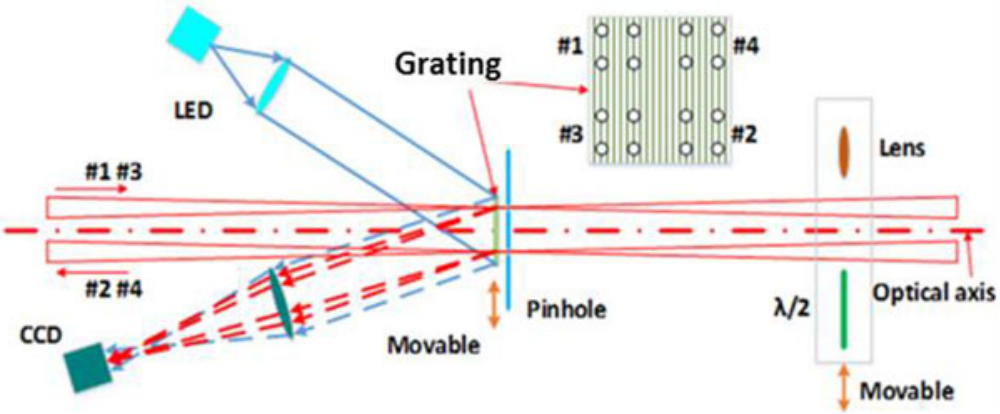

(a)

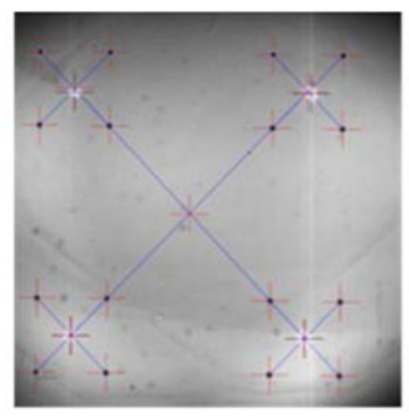

(b)

Figure 19. (a) CSF alignment package and (b) resultant image.
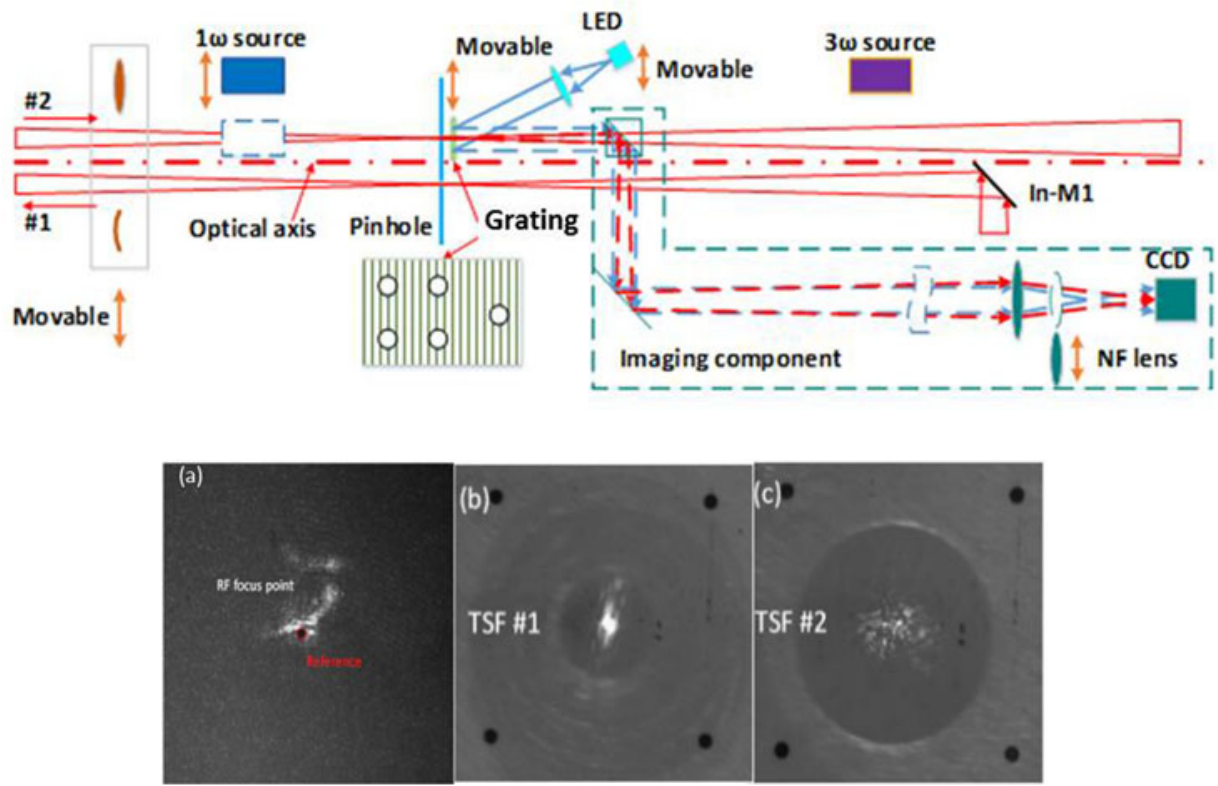

Figure 20. TSF alignment package (top) and result images (bottom): (a) crystals, (b) TSF pass-1 pinhole, and (c) TSF pass-2 pinhole.

for lighting up the grating area to conveniently get the relative position. The half-wave plate acted as the PEPC to realize the four-pass transmission. So the grating, combined with the illumination LED and imaging lens, was used to simultaneously identify the relative positions of the focal points of the four passed laser beams.

The TSF alignment package, shown in Figure 20, was mainly utilized for the three alignment demands: the TSF-1 pinhole, TSF-2 pinhole, and crystals alignment. The package included a set of imaging lenses, grating, cube beam splitter, detector (CCD), and an illumination LED, as well as $1 \omega$ and $3 \omega$ laser sources. The grating provided two far-field references (four symmetric circles) for the two passed laser beams and an additional reference (one circle) for the crystal alignment. For the three references application, the modes were switched by moving the grating, its illumination LED, and part of the imaging component to their preset positions. The $1 \omega$ laser source was mainly applied for the alignment of the optical components in the final optics system and the $3 \omega$ laser source was used for adjusting the target.

(4) Computational optical imaging has been developed for on-shot, near-field, and far-field parameter characterization of high-power lasers ${ }^{[76-78]}$. This method, based on coherent modulation imaging (CMI), has shown significant advantages for high-power lasers, providing the on-shot parameter characterization not only for the phase and intensity of the near field, but also that of the far-field focal spot distribution. It generally has a compact structure and provides a precision measurement capability for the laser field. Figure 21 shows the basic scheme of the high-power laser system, and the diagnostic setup with the CMI method, including the recording devices (CCD1 and CCD2) and pre-characterized random phase plate (RPP), is shown in the box marked in red. Two of the sampling beams were inserted into the measurement package for direct imaging of the far and near fields with CCD3 and CCD4. The complex amplitude of the light field 


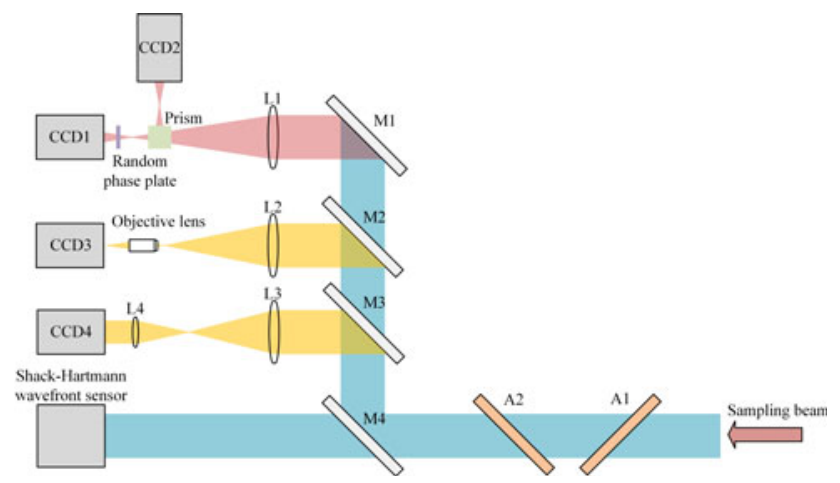

Figure 21. Basic scheme for single-shot beam diagnostics in high-power laser systems with the CMI method.

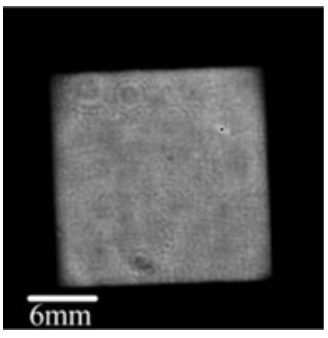

(a)

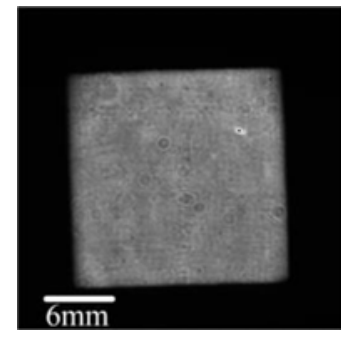

(b)

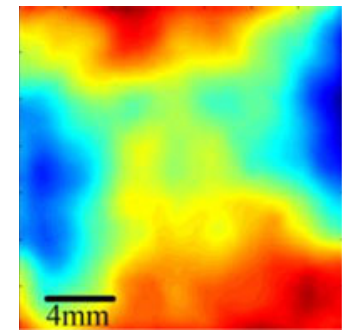

(c)

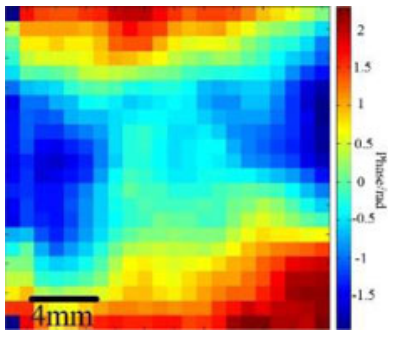

(d)

Figure 22. Comparison of the near-field intensity and phase: (a) near-field intensity reconstructed by the CMI method, (b) near-field intensity measured by direct imaging, (c) near-field phase reconstructed by the CMI method, and (d) near-field phase measured by a Shack-Hartmann wavefront sensor.
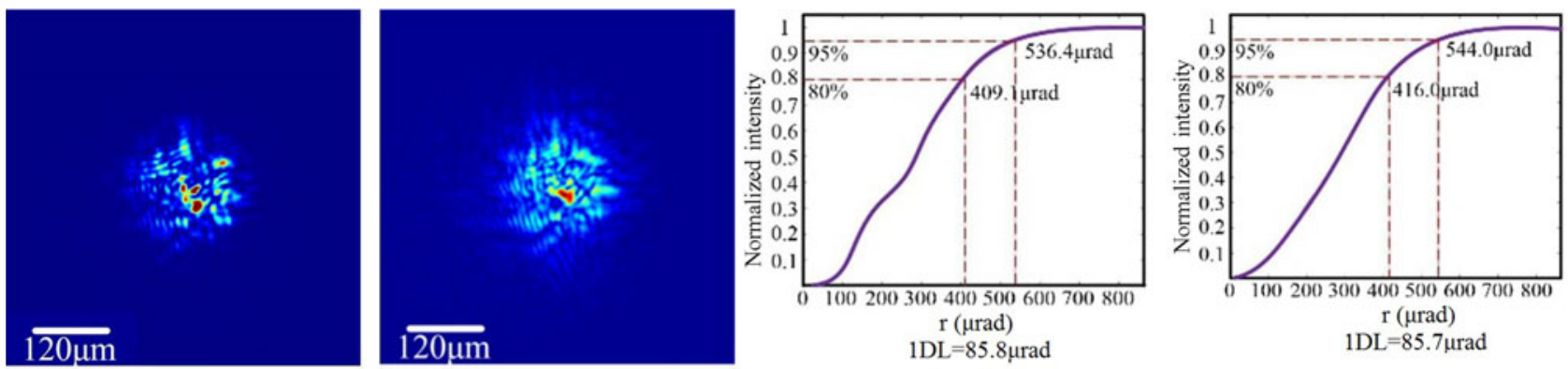

Figure 23. Comparison of the far-field intensity: (a) far-field intensity reconstructed by the CMI method, (b) far-field intensity measured by direct imaging, (c) encircled energy of the far-field focal spots in panel (a), and (d) encircled energy of the far-field focal spot in panel (b).

was reconstructed by iteration calculation using the patterns recorded by CCD1 and CCD2. The near and far fields of the sampling beam were recovered at the same time. The Shack-Hartmann wavefront sensor was used for the nearfield wavefront measurement of low spatial frequency.

Figure 22 shows the comparison of the near-field intensity and phase measured by the CMI method and traditional methods. The output power of the pulsed laser is $3762 \mathrm{~J} / 3 \mathrm{~ns}$. The fill factors of these two methods for the near-field intensity analysis [Figures 22(a) and 22(b)] are 0.723 and 0.702, respectively. From Figures 22(c) and 22(d), there is a significant improvement in the spatial resolution of the phase using the CMI method. Figure 23 shows a comparison of the far-field intensity measured by the CMI method and that by direct imaging. The high dynamic range of the focal spot distribution was obtained by the CMI method in Figures 23(a) and 23(b). Figures 23(c) and 23(d) show the encircled energy of the far-field focal spot corresponding to Figures 23(a) and 23(b), respectively. Using a mathematical analysis, the focal sizes of the $80 \%$ and 95\% encircled energies from the two methods were similar.

(5) The compact FOA was designed and constructed using a wedged focal lens and specific stray light management $^{[79,80]}$. Due to the limited space of the target area, the focal length of the target lens in the SG-II UP laser facility was chosen to be $2.2 \mathrm{~m}$. This relatively short focal lens is shorter than might otherwise be necessary to achieve a fixed harmonic separation, leading to a higher B-integral value for a given laser intensity. Accordingly, it is more 


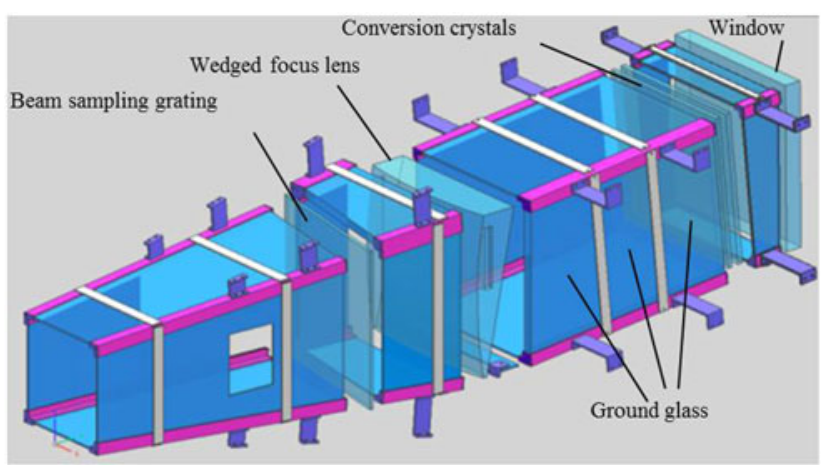

Figure 24. Stray light management by ground glass protection in the FOA.

difficult to manage the stray light and breakup integral in our compact FOA. Two key techniques have been developed to improve the FOA performance in the engineering process including the measurement and adjustment technique of the wedged focus lens, and stray light management technique based on ground glass as shown in Figure 24. The main feature of the stray light management technique is that it consists of a polygon formed by ground and neutral absorbing glass. Therefore, the output energy at $351 \mathrm{~nm}$ of the eight beamlines met the design specifications very well. However, as shown in Figure 7, the high-power laser prototype beam already achieved an energy output up to $16 \mathrm{~kJ} / 5 \mathrm{~ns} / 1 \omega$ and $17.5 \mathrm{~kJ} / 20 \mathrm{~ns} / 1 \omega$. Therefore, there is a significant mismatch between the $1 \omega$ and $3 \omega$ output capabilities. More effort should be concentrated on the improvement of the damage resistance of the final optics system, such as a reduction in the nonlinear propagation effects, improvement of the damage characteristics of large-aperture optics, and avoidance of online contamination with a novel stray light management strategy to increase the operation fluence to an even higher level.

In order to improve the damage threshold of the KDP crystal, the surface qualities of the KDP crystal must be strictly controlled, such as the transmission wavefront, waviness, and roughness. We processed a KDP crystal with ultraprecision single point diamond tools (SPDT). The surface quality results for processing a type II KDP crystal $(350 \mathrm{~mm}$ $\times 350 \mathrm{~mm} \times 10 \mathrm{~mm}$ ) are shown in Figure 25. The surface quality of the transmission wavefront, with $\mathrm{PV}=0.23 \lambda$, RMS PSD1 (in $2.5-33 \mathrm{~mm}$ spatial period band) $=4.8 \mathrm{~nm}$, surface roughness $\mathrm{Rq}=2.33 \mathrm{~nm}$, was similar to that of the U.S. LLNL (RMS (PSD1) $=5 \mathrm{~nm}$, and Rq $=1.5 \mathrm{~nm}$ ). Therefore, the surface quality of the KDP crystal reached a relatively advanced level.

\subsection{SG-II UP PW picosecond system}

The SG-II UP PW picosecond system uses a hybrid technology combining OPCPA and Nd:glass CPA to reduce the pre-pulses and gain narrowing effects. A schematic diagram of the system is shown in Figure 26; the front-end subsystem contains an oscillator, a stretcher, and the OPCPA. A minicompressor with a $100 \mathrm{ps}$ adjustable range is used after the OPCPA to alter the compressed pulse duration and the chirped pulse is injected into the amplifier chain of the SGII 9th laser system. An AO module is utilized to correct the wavefront distortion to produce an optimum focal spot and an off-axis parabolic mirror (OAPM) with $F$-number 2.5 at the end to focus the compressed beam to an extremely high intensity.

The first phase of SG-II UP picosecond laser system development was completed in July 2011 and the system realized laser pulse compression energies of $380 \mathrm{~J} / 5 \mathrm{ps}$ and $370 \mathrm{~J} / 8 \mathrm{ps}$. After obtaining the meter-scale largeaperture grating shown in Figure 27, supported by the joint researching team, an output laser pulse energy $1 \mathrm{~kJ} / 1.7 \mathrm{ps}$ was obtained in September 2016. At the same time, an improved focus spot was obtained, where $50 \%$ of the energy was concentrated in a circle with diameter $20.8 \mu \mathrm{m}$ as shown

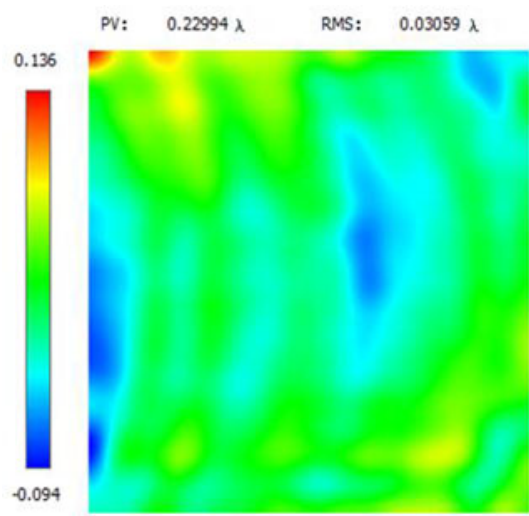

(a)

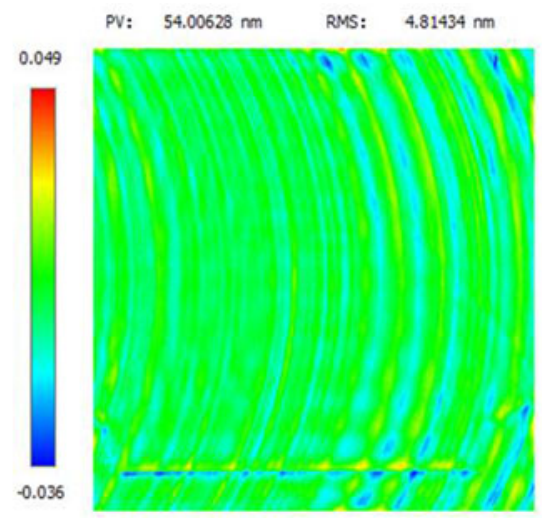

(b)

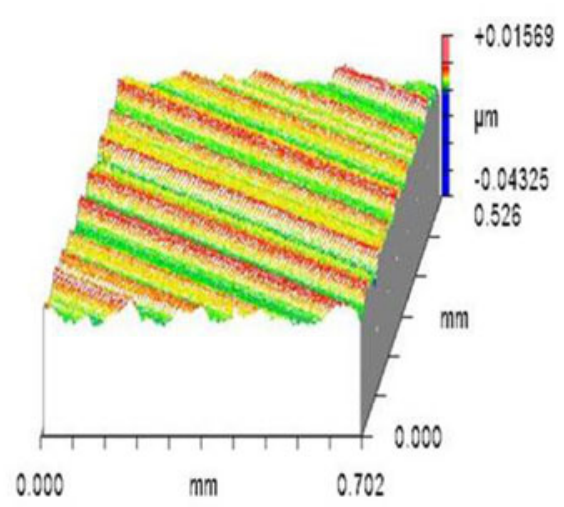

(c)

Figure 25. Results of the surface quality of KDP crystal: (a) wavefront distribution after low-pass filter (spatial period $>3.3 \mathrm{~cm}$ ), (b) wavefront distribution after band passed filter (spatial period $2.5 \mathrm{~mm}-33 \mathrm{~mm}$ ), (c) surface roughness data measured by surface profiler. 


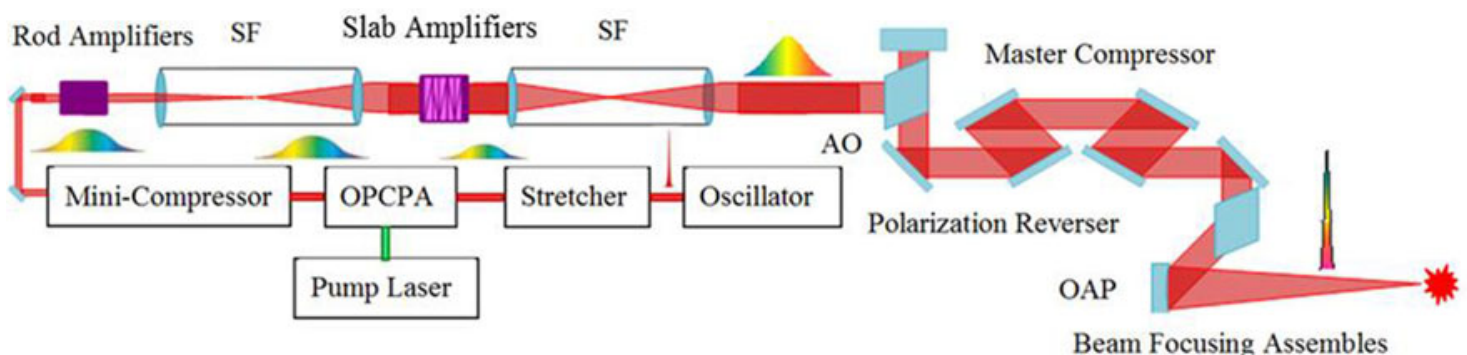

Figure 26. Schematic diagram of the SG-II UP picosecond laser system.

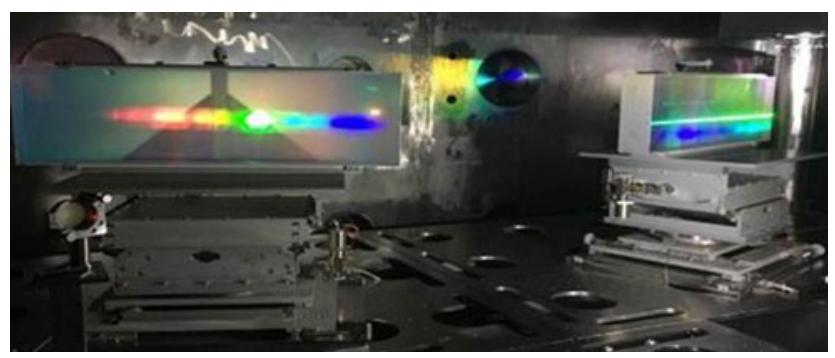

Figure 27. Photograph of large-aperture gratings.

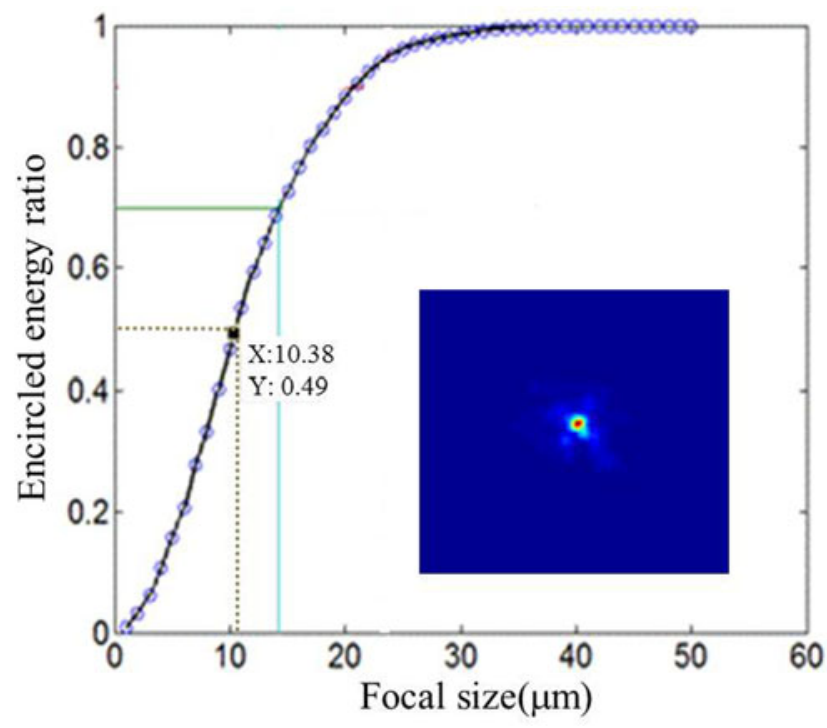

Figure 28. Far field of picosecond laser system.

in Figure 28 detected by a CCD camera ( $100 \mathrm{~J} / 1 \mathrm{ps})$, and the focus power density was estimated to be up to $10^{20} \mathrm{~W} / \mathrm{cm}^{2}$. A pulse contrast of $10^{8}$, obtained before $-81.75 \mathrm{ps}$, was measured with a single-shot cross-correlator as shown in Figure 29 ${ }^{[51]}$ when the pulse width and energy were $0.5 \mathrm{ps}$ and $175 \mathrm{~J}$, respectively.

In the process of the construction of the SG-II UP picosecond laser system, many important technical developments and engineering implementations were achieved. Various problems, such as the high-energy acquisition of pulses with

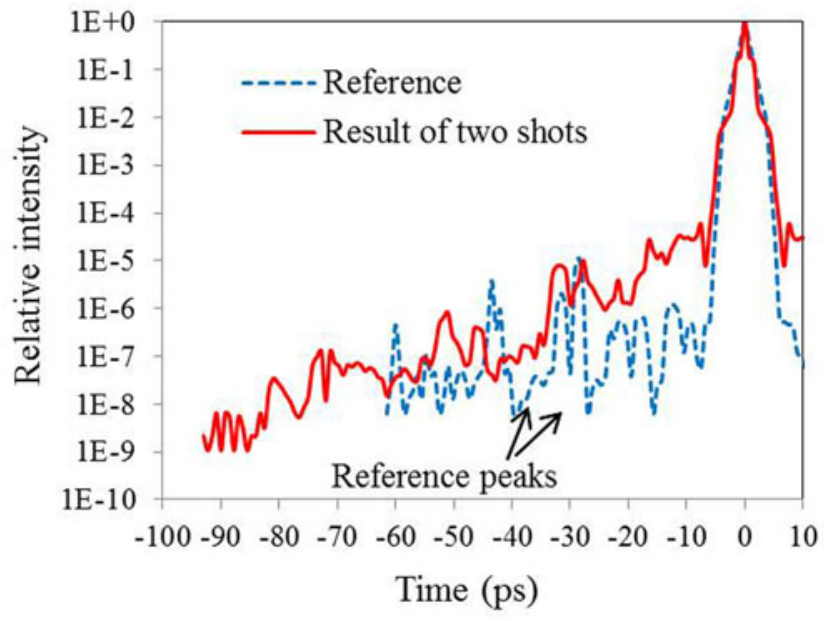

Figure 29. Pulse contrast measurement result of picosecond laser system $^{[50]}$.

wide spectra, the controlling of signal to noise ratio, and focusing and aligning a pulse with a small focal spot size, were solved. Some of these are discussed below.

(1) A stable OPCPA was developed and two-stage OPCPA amplification was employed to amplify chirped pulses to $100 \mathrm{~mJ}$ (gain: $\sim 10^{9}$ ) with pulse spectrum widths of $10 \mathrm{~nm}$. The OPCPA output stability could be controlled within $1 \%$, as shown in Figure 30, mainly benefitting from the high stability pump source and working point design. Through the accumulated operation and assessment, the OPCPA has contributed greatly to the good stability of the whole system, with a $<2 \%$ deviation between the target and actual energy outputs. This contributed to the high success rate of the picosecond laser system ensuring its availability for physical experiments.

(2) Wide spectrum amplification using Nd:glass has been implemented ${ }^{[81]}$, with a design chosen that balanced the output energy and output spectrum, to reduce the B integral accumulated in the system. The shape of the amplified spectrum was adjusted using quartz birefringent filter as the pulse passed through the OPCPA stage and effective broadening of the spectrum was achieved. In comparison with the results without spectral shaping, the spectrum width 


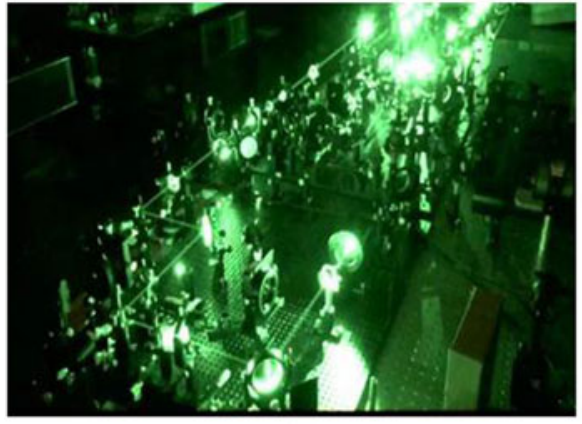

(a)

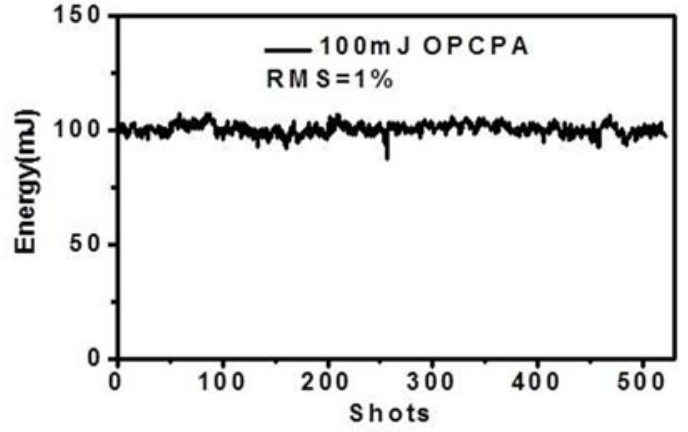

(b)

Figure 30. (a) Photograph of the OPCPA and (b) output energy and stability data of the OPCPA.

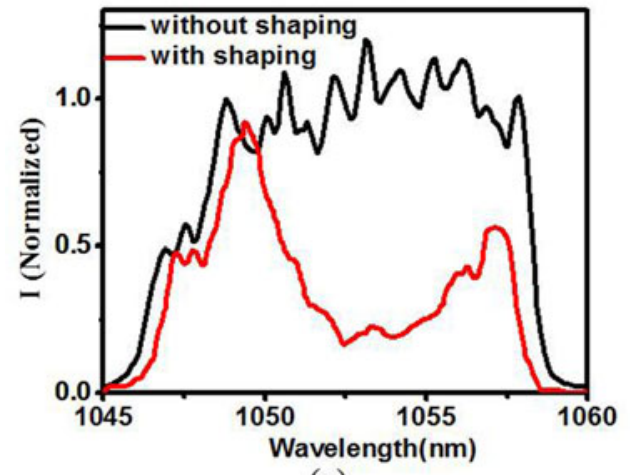

(a)

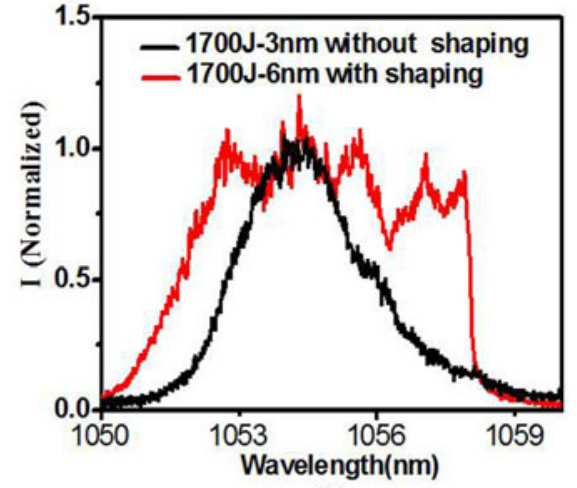

(b)

Figure 31. Comparison of the spectral results before and after shaping: (a) front-end output and (b) main amplifier output.

increased from 3 to $6 \mathrm{~nm}$, as shown in Figure 31.

(3) The petawatt picosecond compression chamber was designed and constructed to provide a clean vacuum environment for the compression gratings. This was important to solve the problem of irreversible damage to the gratings caused when hydrocarbon is ionized and evaporates to the surface of the grating. The petawatt picosecond compression chamber is the largest non-oil vacuum container used today in high-energy picosecond lasers in China. To reduce the volume, the compression chamber was designed as a rectangular structure, with a length of $13 \mathrm{~m}$ and volume of $150 \mathrm{~m}^{3}$. The whole compression chamber was divided into small segments and then the segments were spliced together to improve sealing performance. This method solved the difficulty of processing and transportation. The problem of the cleaning of large vessels was solved by the process of high temperature and pressure water rinsing with a mixed degreasing agent and local high temperature steam cleaning. The problem of high-precision installation of the compression chamber was overcome by digital virtual assembly technology, and the mounting accuracy was up to $2 \mathrm{~mm}$. Figure 32(a) shows exterior of the compression chamber. Figure 32(b) illustrates the layout of the pulse compressor where four tiled multilayer dielectric (MLD) gratings ${ }^{[82]}$ with 1740 line/mm were employed. Among the four gratings, the last grating G4 was more easily damaged. The intrinsic damage of the gratings took the form of pinpoints, as shown in Figure 33(a). The grating structures were destroyed with multiple shots. The damage depth was only limited within the first several dielectric layers. The damage growth threshold is lower than the intrinsic damage threshold. Once damage was initialized, the damage area grows linearly with shots, as shown in Figure 33(b). The intensity modulation of the damage site plays a major role in damage growth. The damage growth and large-area damage region greatly reduce the diffraction efficiency. The damage resistance of the compressor grating is still a key issue for high-energy operation of petawatt laser systems.

(4) Adaptive optics has been adopted in the petawatt system. A primary requirement for a petawatt picosecond laser chain is that $50 \%$ of the design energy should be contained within a $25-\mu \mathrm{m}$ focal spot at the target plane. This is approximately four times that of the diffraction-limited $80 \%$ energy spot size. To meet the spot size requirement and goal, an AO setup was adopted to correct for wavefront aberrations. The lower-order aberrations could be classified and effectively reduced with this AO setup. A 52-actuator large-aperture deformable mirror (clear aperture $\Phi 38 \mathrm{~cm}$ ), 


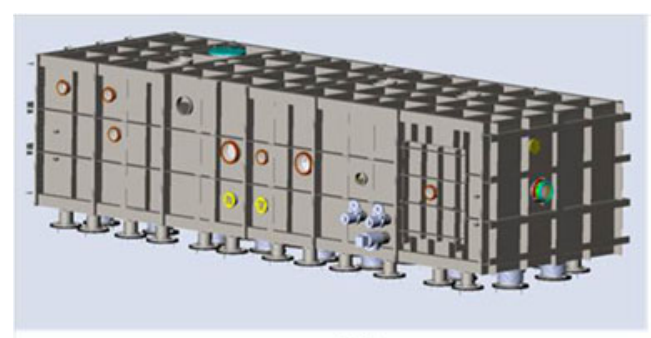

(a)

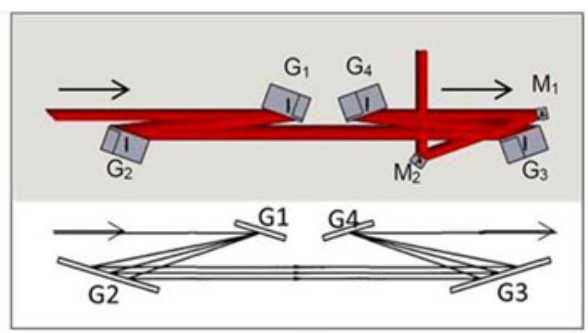

(b)

Figure 32. (a) Exterior of the compression chamber and (b) layout of the pulse compressor (plan view); G1, G2, G3, and G4 are the tiled MLD gratings and $\mathrm{M} 1$ and M2 are the mirrors; the light passes through G1, G2, G3, and G4 sequentially (arrow direction).

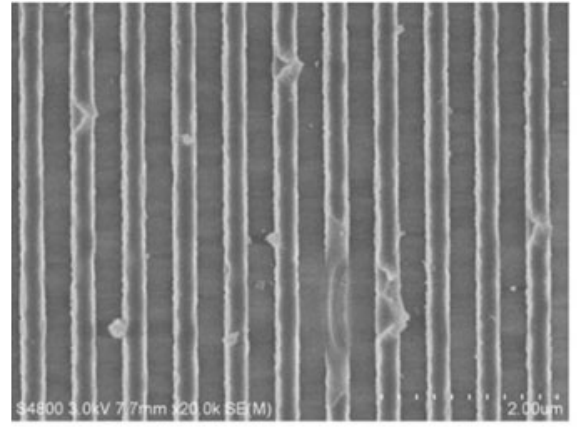

(a)

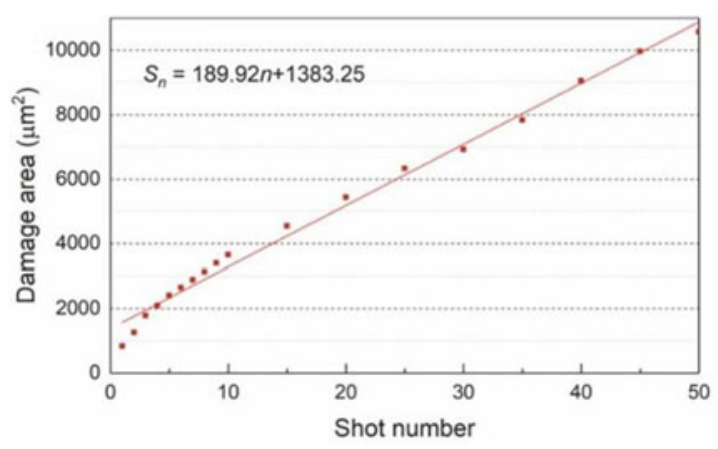

(b)

Figure 33. Picosecond laser damage of gratings. (a) Typical morphology of pinpoint damages on the grating and (b) linear damage area growth with shot number.

shown in Figure 34, was placed at the end of the main amplifier. At the compressor output, a pick-off mirror sends the sampled beam through a relay imaging system to a Shack-Hartmann sensor comprised of an array of $22 \times 22$ lenslets $(35 \mu \mathrm{m} \times 35 \mu \mathrm{m}$ square size for each lenslet). An OPCPA seed laser was used as the sample beam to correct the static aberrations, including the misalignment error and manufacturing defects. This enables the laser chain to produce a wavefront error within $1 \lambda(\mathrm{PV})$. The pump induced wavefront error was also measured and corrected to ensure the best performance of the system.

(5) A target alignment system was established to satisfy the specifications. The petawatt laser auxiliary alignment system (AAS) is shown in Figure 35. This consists of a largeaperture telescope comprising an achromatic lens, OAPM, illumination source, a movable plate, and a beam splitter. For the fast ignition experimental shot, a solid gold cone was positioned at the center of the cylindrical hohlraum target, oriented in the same direction as the petawatt laser, perpendicular to the nanosecond laser entrance holes. The telescope was used to monitor both the focus spot of the petawatt laser and center of the golden cone. During the alignment process, a movable standard plate with a parallelism of $2 \mu \mathrm{rad}$ was moved into the petawatt laser path. The alignment procedure was set to ensure smooth operation. The petawatt laser pointing error was tested through several

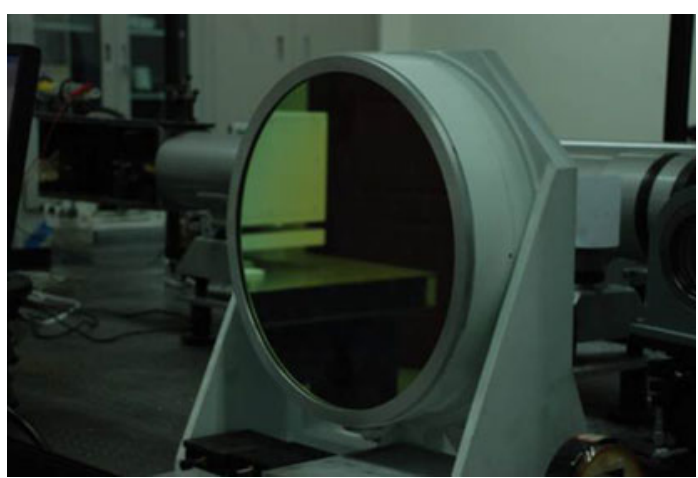

Figure 34. Deformable mirror of the AO setup for the petawatt picosecond laser chain.

shots and the recorded results of the offset of the petawatt beam centroids to the net crossings demonstrated that the total pointing error was $9.76 \mu \mathrm{m}$ (RMS). This is less than the required $10 \mu \mathrm{m}$ (RMS) for the detailed description of the target alignment system (see Ref. [83]).

(6) An apparatus to measure the pulse contrast with a high dynamic range has been developed. The apparatus ${ }^{[50]}$ was based on the single-shot cross-correlation with a fiber array detector. A schematic diagram of the pulse contrast measurement is shown in Figure 36. Cross-correlation has 


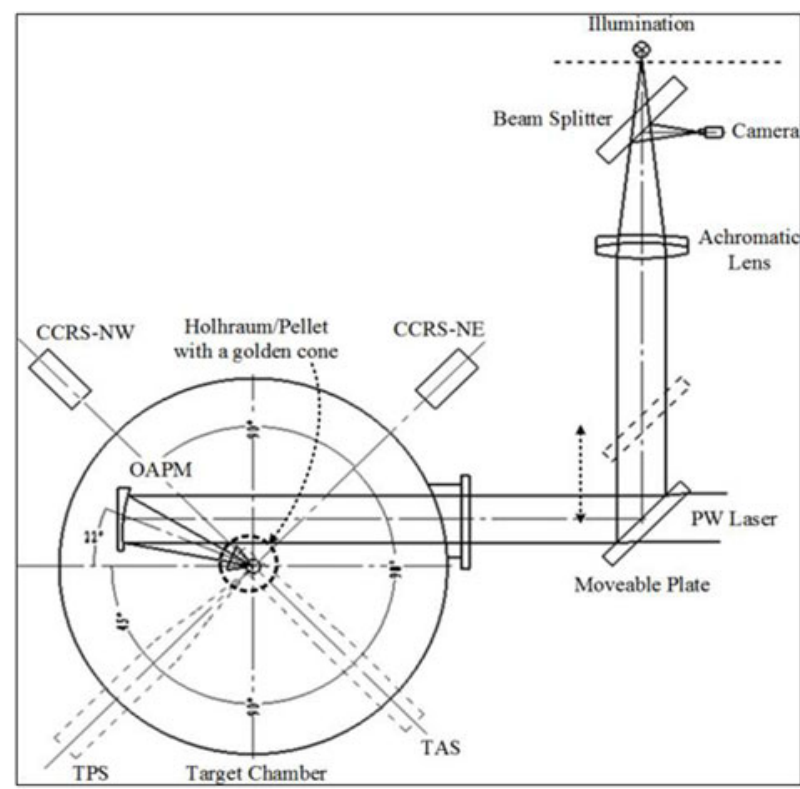

Figure 35. Laser auxiliary alignment system. CCRS-NW: northwest chamber center reference system; CCRS-NE: northeast chamber center reference system; TAS: target alignment sensor; TPS: target positioning system; OAPM: off-axis parabola mirror ${ }^{[83]}$.

advantages for the pulse contrast measurement over autocorrelation because it identifies noise before or after main pulse. The fiber array was used to convert the spatial signal into a series of temporal signals so that the signal series could be conveniently analyzed by a set of PMTs (photomultiplier tubes) and an oscilloscope. A beam splitter placed after the attenuator, $A_{1}$, divided the sampling beam into two parts. The transmitted part as the measured pulse was directed to a cross-correlation generation crystal (XCGC) through a mirror, $\mathrm{M}_{\mathrm{X} 1}$, and a cylindrical lens, $\mathrm{L}_{1}$. The reflected part as a grating beam went through a second harmonic generation crystal (SHGC) to produce a $2 \omega$ pulse. The $2 \omega$ pulse subsequently passed through $\mathrm{M}_{\mathrm{X} 2}, \mathrm{M}_{\mathrm{X} 3}, \mathrm{M}_{\mathrm{X} 4}$, and the cylindrical lens, $\mathrm{L}_{2}$, before arriving at the XCGC. After the attenuator, $\mathrm{A}_{2}$, a cylindrical lens, $\mathrm{L}_{3}$, behind the XCGC coupled the single-shot cross-correlation signal into the fiber array detector and the detector's sensitivity was improved because of the fiber array and $\mathrm{PMT}^{[84]}$. The dynamic range of this measurement was $>10^{8}$ due to the fine attenuators, such as the filters $\left(A_{1}\right)$, coated mirrors $\left(A_{2}\right)$, and fiber attenuators $\left(\mathrm{A}_{3}\right)$. The removable uncoated parallel plates, $\mathrm{P}_{1}, \mathrm{P}_{2}$, and $\mathrm{P}_{3}$ could be used for identifying errors due to the saturation of the cross-correlation and calibration. The pulse contrast results of Figure 29 were obtained with this apparatus.

\section{Recent development of SG-II 5 PW facility}

The SG-II 5 PW facility is under construction and aims to produce $150 \mathrm{~J} / 30 \mathrm{fs}$ pulses centered at $808 \mathrm{~nm}$. This facility

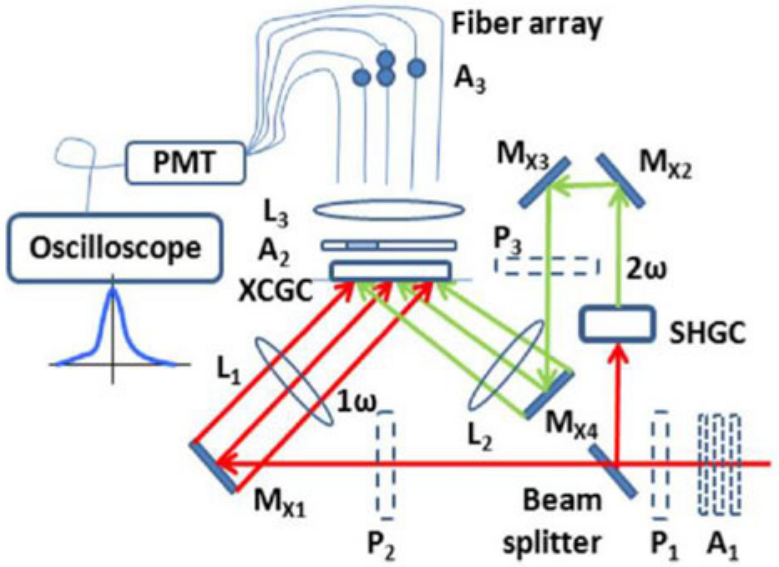

Figure 36. Schematic diagram of the pulse contrast measurement. $\mathrm{M}_{\mathrm{X} 1}$, $\mathrm{M}_{\mathrm{X} 2}, \mathrm{M}_{\mathrm{X} 3}$, and $\mathrm{M}_{\mathrm{X} 4}$ are mirrors; $\mathrm{P}_{1}, \mathrm{P}_{2}$, and $\mathrm{P}_{3}$ are removable parallel plates; $\mathrm{L}_{1}, \mathrm{~L}_{2}$, and $\mathrm{L}_{3}$ are cylindrical lenses; $\mathrm{A}_{1}, \mathrm{~A}_{2}$, and $\mathrm{A}_{3}$ are attenuators; SHGC is the autocorrelation generation crystal; XCGC is the crosscorrelation generation crystal; and PMT is the photomultiplier tube.

can provide a powerful tool for fundamental and frontier physics. As shown in Figure 37, the SG-II 5 PW facility is completely based on a three-stage non-collinear OPCPA. A detailed description with the technical progress of the SG-II 5 PW system is given in Ref. [85]. All stages are designed for compressed pulses of different power levels for various utilizations. At the first stage amplifier, pulses of 1-10 TW at a repetition of $1 \mathrm{~Hz}$ have been generated with an existing pump laser based on Nd:YAG. At the second stage amplifier, pulses of $1 \mathrm{PW}$ level are produced with the 7th beam of the SG-II facility as a pump source that operates one shot per hour. The third stage, as the master amplifier, is under construction and designed to deliver pulses of $5 \mathrm{PW}$ using the SG-II 9th beam as a pump laser. This third stage may be completed in two years. For the master amplifier at present, an LBO crystal with a $15 \mathrm{~cm} \times 15 \mathrm{~cm}$ aperture is demanded to boost the energy of chirped pulses higher than $250 \mathrm{~J}$ and spectrum bandwidth $>40 \mathrm{~nm}$. After the amplifiers, the beam is expanded to dimensions of $29 \mathrm{~cm} \times$ $29 \mathrm{~cm}$ using a spatial filter (SF5) and injected into the $\mathrm{AO}$ system for the wavefront measurements and correction. The chirped pulse of the fine beam quality is compressed by a gold-coated-grating-based compressor and a pulse of $150 \mathrm{~J}$ with a 30 -fs duration is generated. The compressed pulse is delivered into the focusing system consisting of an OAPM and related detecting elements. The reflected OAPM is applied to realize a smaller focal spot, higher intensity $\sim 10^{21} \mathrm{~W} / \mathrm{cm}^{2}$, and better energy concentration (50\% energy within a $2 \times$ diffraction-limited spot size). At this intensity level a pulse contrast of $>10^{8}$ is required to meet the demands of high field experiments. Moreover, supporting systems, such as collimation, measurement, and controlling have been developed for routine operations.

As illustrated in Section 2, at present, we have completed two stages of the OPCPA amplifiers. A chirped pulse of 


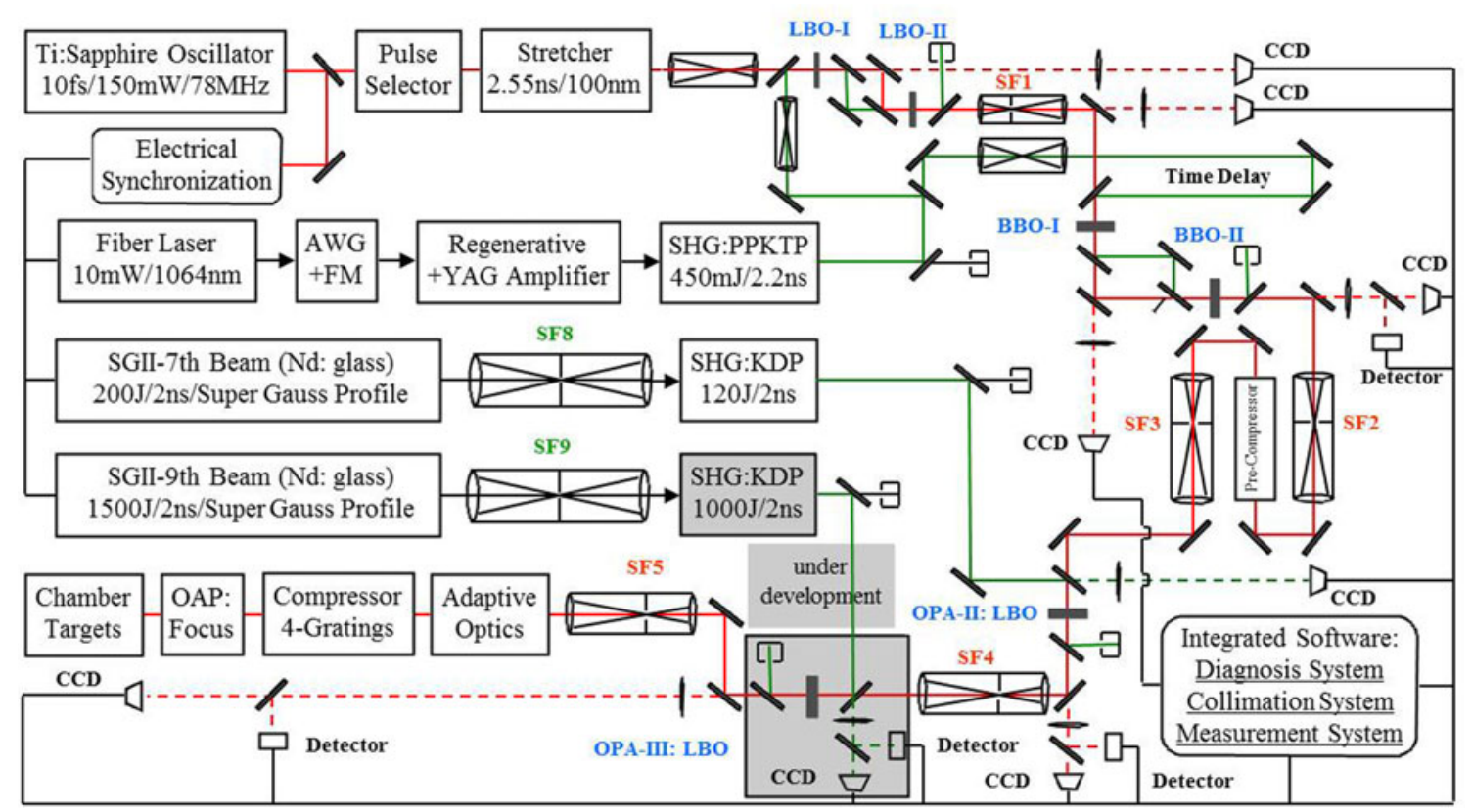

Figure 37. Schematic diagram of the SG-II 5 PW laser facility; OAPM: off-axis parabolic mirror, FM: frequency modulator, and AWG: arbitrary waveform generator.

49.7 $\mathrm{J}$ and a FWHM spectrum bandwidth of $85 \mathrm{~nm}$ were obtained. The highest conversion efficiency of up to $41.9 \%$ was experimentally demonstrated for the first time in high-energy petawatt-class OPCPAs. The total transmission efficiency of the compressor was $\sim 75 \%$ and energy of the compressed pulse was higher than $37 \mathrm{~J}$. As shown in Figure 38, traces of the compressed pulse were measured with the whole beam diameter by an autocorrelator and the temporal duration was $21 \mathrm{fs}$ (FWHM). A peak power of up to $1.76 \mathrm{PW}$ has been obtained after the second OPCPA stage. The focal spot size imaged by the CCD indicates a $10 \mu \mathrm{m}$ diameter with closed-loop correction by the AO system without the OPCPA operation (Figure 39(a)).

In 2017, the SG-II 5 PW facility became operational and was utilized for high-energy physics experiments. The primary results demonstrated that laser proton accelerations of $>7 \mathrm{MeV}$ on average and $10 \mathrm{MeV}$ maximum were achieved for a focused power density exceeding $10^{19} \mathrm{~W} / \mathrm{cm}^{2}$. The focal spot imaged online by an X-ray pinhole camera is presented in Figure 39(b). This saturated image demonstrates that the spot size at the target was smaller than $45 \mu \mathrm{m}$ in diameter.

We have developed integrated software with a userfriendly interface to implement diagnostics, collimation, and measurement so that the real-time data, including the status of affairs (date, time, serial number, and abnormal operation information of every shot), amplified pulse energy, spectrum, pulse waveform, near field, and far field were automatically collected, processed, and stored. In both the pump and signal laser beams of all of the amplifiers, at the incident and output positions auto-collimation systems including the CCD and

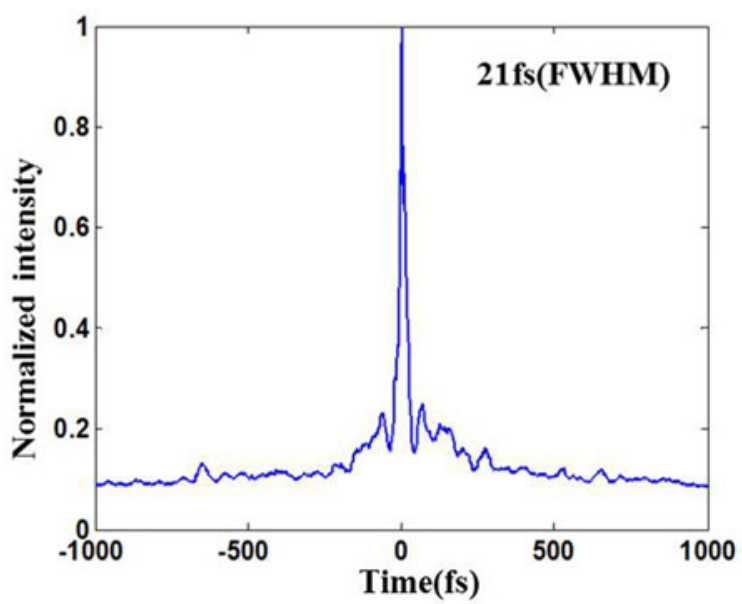

Figure 38. Compressed pulse duration with the whole beam diameter.

detectors were installed, which ensured the high OPCPA conversion efficiency and long optical path transmission. The pulse width after compression was monitored by the home-made full-beam single-shot autocorrelator and accurately controlled by a mini-compressor after OPCPAI. The mini-compressor not only compensated the residual dispersion between the stretcher and main compressor, but also eliminated the parametric fluorescence from the OPCPA, improving the temporal contrast of the pulse. The spatial filter designs were based on the image relay for beam expansion, high spatial frequency noise filter, and amplified optical parametric fluorescence restriction. For 


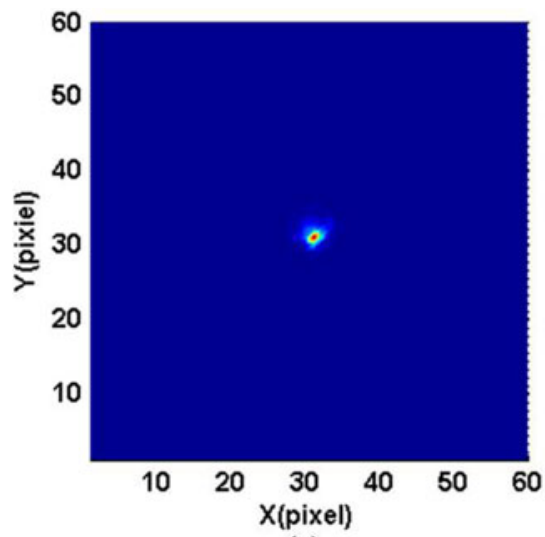

(a)

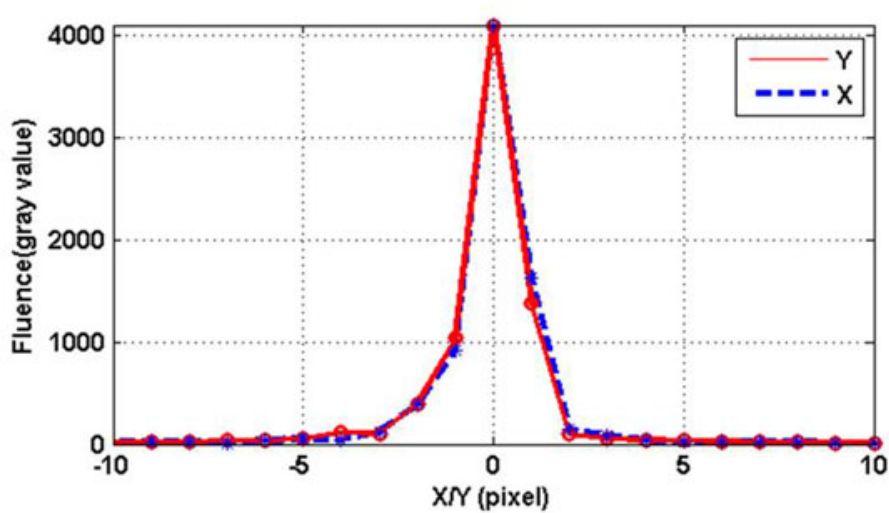

(b)

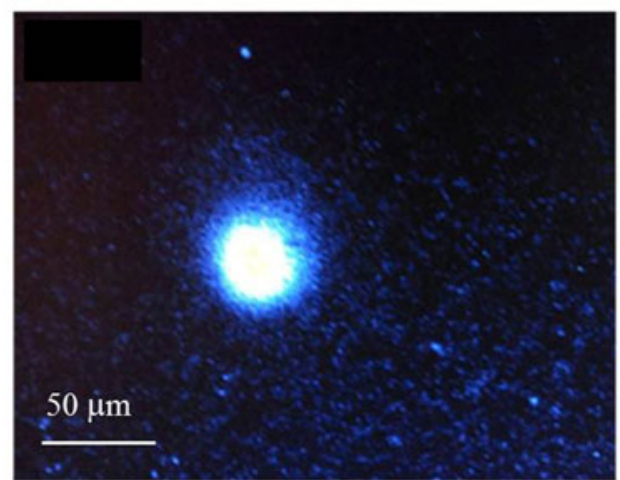

(c)

Figure 39. (a) Measured focal spot imaged by the CCD after AO correction without optical parametric amplification, (b) horizontal and vertical line-outs of focal spot image, and (c) focal spot imaged by an X-ray pinhole camera in the high-energy experiments.

better routine operation, each spatial filter was equipped with a computer-controlled auto-collimation system with actively driven mirrors and an online measurement unit. However, the use of large-aperture refractive spatial filters in a broadband ultra-short pulse laser system could introduce considerable chromatic aberration resulting in degradation of the spatiotemporal quality of the output pulse ${ }^{[86]}$. Hence a chromatic aberration compensator has been designed and will be inserted after OPCPA-I to solve this issue.

At the beginning of 2018, the latest outline of work has been optimized for the first stage of the OPCPA so that its performance has been greatly improved. The prior two LBO crystals (LBO-I and LBO-II) were replaced by BBO crystals with a parametric bandwidth of $\sim 100 \mathrm{~nm}$, and the beam diameters of both the pump and signal were correspondingly expanded to $4 \mathrm{~mm}$ to reduce the effects of spatially coupled near-field drift due to environmental vibration. By numerical simulations, the lengths of all four BBO crystals, as well as the pump energy allocations for each stage, were optimized. With pump pulses of $390 \mathrm{~mJ}$, the sub-amplifiers based on BBO-III and BBO-IV have delivered signal pulse energy of $160 \mathrm{~mJ}$, indicating a pump-to-signal conversion efficiency of $\sim 41 \%$. The total net gain of the first stage of the OPCPA

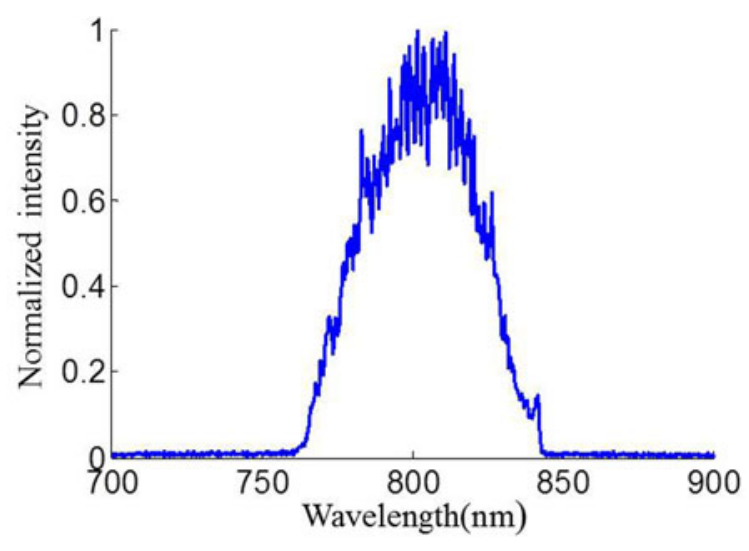

Figure 40. Spectrum of the first OPCPA stage.

has a maximum value of $8 \times 10^{8}$, and the bandwidth was improved from 62 to $80 \mathrm{~nm}$ (FWHM) (see Figure 40). The spectral profile indicates that the amplification for the last BBO crystal worked in an unsaturated region that is useful for limiting the optical parametric fluorescence ${ }^{[78]}$. As shown in Figure 41, the stability of the first OPCPA was $\sim 1.67 \%$ RMS, corresponding to a pump laser stability of $1 \%$ 


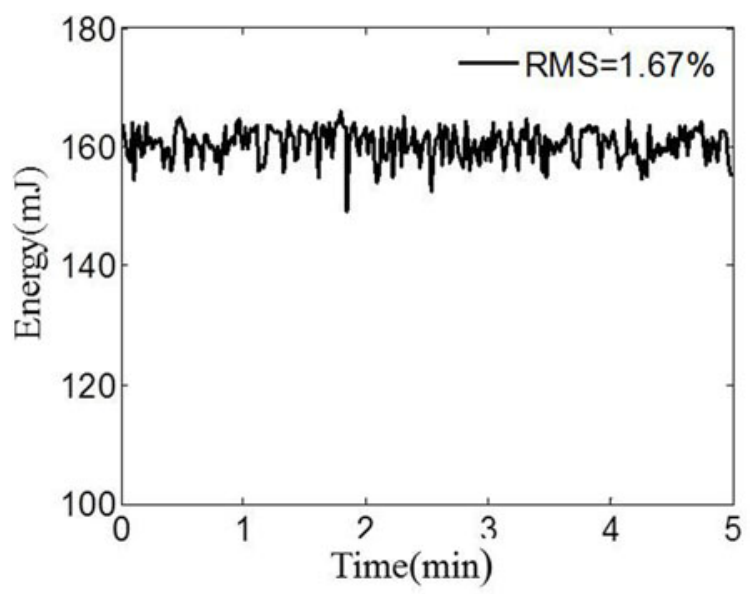

Figure 41. Energy and stability of signal pulses from the first OPCPA stage.

RMS. The PW-scale amplifier is expected to benefit from the improvement of signal pulses from OPCPA-I.

\section{Summary}

The multifunctional platform including a series of highpower laser facilities at NLHPLP is reviewed. The platform has played an important role in the ICF and HED research fields. The SG-II series facilities available to international users can be operated independently or in combination, with the capability of a wide range of pulse widths. As a newly built facility, the SG-II UP facility, consisting of a single petawatt picosecond system and eight-beam nanosecond system, has achieved the required outputs, and several key technologies have been developed to ensure the performance of the facility. In addition, various physical experiments have been performed with the SG-II 5 PW facility. It is expected that the running platform will continue to provide experimental shots for ICF and HED research. The NLHPLP will continue to enhance the abilities of designing, constructing, and operating high-power facilities, enabling possible larger-scale laser drive developments. Meanwhile, in view of the present large challenges in achieving ignition, we will further improve the openness of our facilities to improve international cooperation.

\section{Acknowledgements}

We would like to thank the institutions and individuals who have provided useful discussions and assistance to the NLHPLP. This work was partially supported by International Partnership Program of Chinese Academy of Sciences (No. 181231 KYSB20170022). And this work is also partially supported by the Key Projects of International Cooperation in Chinese Academy of Sciences.

\section{References}

1. J. Nuckolls, L. Wood, A. Theissen, and G. Zimmerman, Nature 239, 139 (1972).

2. J. L. Emmett, J. Nuckolls, and L. Wood, Sci. Amer. 230, 24 (1974).

3. J. F. Holzrichter, Nature 316, 309 (1985).

4. C. A. Haynam, P. J. Wegner, J. M. Auerbach, M. W. Bowers, S. N. Dixit, G. V. Erbert, G. M. Heestand, M. A. Henesian, M. R. Hermann, K. S. Jancaitis, K. R. Manes, C. D. Marshall, N. C. Mehta, J. Menapace, E. Moses, J. R. Murray, M. C. Nostrand, C. D. Orth, R. Patterson, R. A. Sacks, M. J. Shaw, M. Spaeth, S. B. Sutton, W. H. Williams, C. C. Widmayer, R. K. White, S. T. Yang, and B. M. Van Wonterghem, Appl. Opt. 46, 3276 (2007).

5. P. Vivini and M. Nicolaizeau, Proc. SPIE 9345, 934503 (2015).

6. Private communication in the 2nd International symposium on High Power Laser Science and Engineering (HPLSE) in 2016.

7. X. T. He and W. Y. Zhang, Eur. Phys. J. D 44, 227 (2007).

8. X. M. Deng and W. Y. Yu, IEEE J. Quant. Electron. 17, 1650 (1981).

9. X. T. He, X. M. Deng, D. Y. Fan, X. M. Zhang, Z. Q. Lin, N. Y. Wang, Z. J. Zheng, and J. R. Liu, Fusion Eng. Design 44, 57 (1999).

10. Z. Q. Lin, X. M. Deng, D. Y. Fan, S. J. Wang, S. H. Chen, J. Q. Zhu, L. J. Qian, X. H. Shen, F. M. Xu, J. Zhu, W. X. Ma, X. L. Xie, Y. X. Zheng, W. Q. Zhang, Q. H. Chen, M. Y. Ling, H. Y. Huang, and J. Z. Zhang, Fusion Eng. Design 44, 61 (1999).

11. G. Y. Xiao, D. Y. Fan, S. J. Wang, Z. Q. Lin, Y. Gu, J. Q. Zhu, Y. X. Zhen, J. Zhu, F. Q. Liu, S. H. Chen, Q. H. Chen, G. L. Huang, and X. M. Deng, Third International Conference on Solid State Lasers for Application to Inertial Confinement Fusion (1999), p. 890.

12. Z. Q. Lin, S. J. Wang, D. Y. Fan, Y. Gu, Z. J. Zheng, J. Q. Zhu, J. Zhu, X. J. Cai, G. L. Huang, Y. P. Dai, and Y. Yang, High Power Laser Part. Beams 14, 403 (2002).

13. Y. Zhao, Proc. SPIE 3047, 54 (1997).

14. J. Zhu, Chin. J. Nature 28, 271 (2006).

15. X. T. He, J. Phys. Conf. Ser. 688, 012029 (2016).

16. G. Xu, T. Wang, Z. Li, Y. Dai, Z. Lin, Y. Gu, and J. Zhu, Rev. Laser Eng. Suppl. 36, 1172 (2008).

17. Y. Gao, Z. Cao, X. Yang, W. Ma, B. Zhu, and Z. Lin, in Conference on Lasers and Electro-Optics Pacific Rim (2015), paper 25D2_3.

18. J. Q. Zhu, J. Zhu, X. Li, B. Zhu, W. Ma, D. Liu, C. Liu, X. Lu, W. Fan, Z. Liu, D. Zhao, S. Zhou, Y. Zhang, L. Wang, M. Sun, B. Wang, Z. Jiao, L. Ren, G. Zhang, J. Miao, and Z. Lin, Proc. SPIE 10084, 1008405 (2017).

19. X. Xie, J. Zhu, Q. Yang, J. Kang, H. Zhu, A. Guo, P. Zhu, and Q. Gao, Proc. SPIE 9513, 95130A (2015).

20. http://sglf.siom.ac.cn/UserFacility/AnnualPlan and http://lssf. cas.cn/dzzRegisterController.do?outerelss\&flag=99.

21. H. S. Peng, Z. J. Zheng, B. H. Zhang, Y. K. Ding, Y. J. Tang, Y. M. Zheng, S. H. Wen, Z. L. Liu, Y. N. Ding, D. Y. Tang, J. Feng, J. B. Chen, J. X. Cheng, W. Y. Mao, S. Y. Lui, S. E. Jiang, L. Y. Qi, L. H. Qiu, S. J. Yao, H. P. Xu, P. Q. Luo, R. Yu, Y. H. Zhang, G. N. Chen, D. X. Lai, S. P. Zhu, W. Y. Zhang, T. Q. Chang, and X. T. He, J. Fusion Energy 19, 81 (2000).

22. J. W. Yang, T. S. Li, T. Yi, C. K. Wang, M. Yang, W. M. Yang, S. Y. Liu, S. E. Jiang, and Y. K. Ding, Fusion Sci. Technol. 72, 41 (2017).

23. J. Zhong, Y. Li, X. Wang, J. Wang, Q. Dong, C. Xiao, S. Wang, X. Liu, L. Zhang, L. An, F. Wang, J. Zhu, Y. Gu, X. He, G. Zhao, and J. Zhang, Nat. Phys. 6, 984 (2010).

24. H. Shu, S. Fu, X. Huang, J. Wu, H. Zhou, and J. Ye, Meas. Sci. Technol. 23, 015203 (2012). 
25. Y. Gu, F. Zhang, L. Shan, B. Bi, J. Chen, L. Wei, J. Li, Z. Song, Z. Liu, Z. Yang, M. Yu, B. Cui, Y. Zhang, H. Liu, D. Liu, W. Wang, Z. Dai, Y. Yang, L. Yang, F. Zhang, X. Wu, K. Du, W. Zhou, L. Cao, B. Zhang, J. Wu, G. Ren, H. Cai, S. Wu, L. Cao, H. Zhang, C. Zhou, and X. He, High Power Laser Part. Beams 27, 110101 (2015).

26. X. T. He, H. B. Cai, S. Z. Wu, L. H. Cao, H. Zhang, M. Q. He, M. Chen, J. F. Wu, C. T. Zhou, W. M. Zhou, L. Q. Shan, W. W. Wang, F. Zhang, B. Bi, Z. Q. Zhao, Y. Q. Gu, B. H. Zhang, W. Wang, Z. H. Fang, A. L. Lei, C. Wang, W. B. Pei, and S. Z. Fu, Plasma Phys. Control. Fusion 57, 064003 (2015).

27. Y. J. Rhee, S. M. Nam, J. Peebles, H. Sawada, M. Wei, X. Vaisseau, T. Sasaki, L. Giuffrida, S. Hulin, B. Vauzour, J. J. Santos, D. Batani, H. S. McLean, P. K. Patel, Y. T. Li, D. W. Yuan, K. Zhang, J. Y. Zhong, C. B. Fu, N. Hua, K. Li, Y. Zhang, J. Q. Zhu, I. J. Kim, J. H. Jeon, T. M. Jeong, I. W. Choi, H. W. Lee, J. H. Sung, S. K. Lee, and C. H. Nam, Laser Part. Beams 34, 645 (2016).

28. D. Yuan, Y. Li, M. Liu, J. Zhong, B. Zhu, Y. Li, H. Wei, B. Han, X. Pei, J. Zhao, F. Li, Z. Zhang, G. Liang, F. Wang, S. Weng, Y. Li, S. Jiang, K. Du, Y. Ding, B. Zhu, J. Zhu, G. Zhao, and J. Zhang, Sci. Rep. 7, 42915 (2017).

29. T. Q. Chang, Y. K. Ding, D. X. Lai, T. X. Huan, S. P. Zhu, Z. J. Zheng, G. Y. Wang, Y. M. Zheng, X. T. He, W. B. Pei, Q. S. Duan, W. Y. Zhang, T. G. Feng, G. N. Chen, and P. J. Gu, Phys. Plasmas 9, 4744 (2002).

30. W. Y. Zhang and X. T. He, J. Phys. Conf. Ser. 112, 032001 (2008).

31. Y. T. Yuan, L. F. Wang, S. Y. Tu, J. F. Wu, Z. R. Cao, X. Y. Zhan, W. H. Ye, S. Y. Liu, S. E. Jiang, Y. K. Ding, and W. Y. Miao, Acta Phys. Sin. 63, 235203 (2014).

32. L. Jing, H. Li, Z. Lin, L. Li, L. Kuang, Y. Huang, L. Zhang, T. Huang, S. E. Jiang, and Y. Ding, Plasma Sci. Technol. 17, 842 (2015).

33. Q. L. Dong, S. J. Wang, Q. M. Lu, C. Huang, D. W. Yuan, X. Liu, X. X. Lin, Y. T. Li, H. G. Wei, J. Y. Zhong, J. R. Shi, S. E. Jiang, Y. K. Ding, B. B. Jiang, K. Du, X. T. He, M. Y. Yu, C. S. Liu, S. Wang, Y. J. Tang, J. Q. Zhu, G. Zhao, Z. M. Sheng, and J. Zhang, Phys. Rev. Lett. 108, 215001 (2012).

34. D. Yuan, J. Wu, Y. Li, X. Lu, J. Zhong, C. Yin, L. Su, G. Liao, H. Wei, K. Zhang, B. Han, L. Wang, S. Jiang, K. Du, Y. Ding, J. Zhu, X. He, G. Zhao, and J. Zhang, Astrophys. J. 815, 46 (2015).

35. Y. Zhu, Q. Su, G. Chen, and L. Dai, Sci. Technol. Rev. 3, 30 (2012).

36. D. Pile, Nat. Photon. 5, 644 (2011).

37. H. Shu, X. Huang, H. Pan, J. Ye, F. Zhang, G. Jia, Z. Fang, Y. Tu, Z. Xie, and S. Fu, Int. J. Fract. 206, 81 (2017).

38. Y. Zhao, J. Yang, J. Zhang, G. Yang, M. Wei, G. Xiong, T. Song, Z. Zhang, L. Bao, B. Deng, Y. Li, X. He, C. Li, Y. Mei, R. Yu, S. Jiang, S. Liu, Y. Ding, and B. Zhang, Phys. Rev. Lett. 111, 155003 (2013).

39. S. E. Jiang, Y. K. Ding, S. Y. Liu, B. H. Zhang, Z. J. Zheng, J. M. Yang, W. Y. Miao, T. X. Huang, J. Y. Zhang, S. W. Li, and L. F. Cao, Wuli 39, 531 (2010).

40. W. L. Shang, W. H. Zhang, R. Z. Yu, T. Zhu, F. Wang, J. M. Yang, S. Y. Liu, and S. E. Jiang, Phys. Plasmas 24, 072707 (2017).

41. J. Yang, T. Li, T. Yi, C. Wang, M. Yang, W. Yang, S. Liu, S. Jiang, and Y. Ding, Plasma Sci. Technol. 18, 1044 (2016).

42. C. Danson, D. Hillier, N. Hopps, and D. Neely, High Power Laser Sci. Eng. 3, e3 (2015).

43. L. Q. Shan, H. B. Cai, W. S. Zhang, Q. Tang, F. Zhang, Z. F. Song, B. Bi, F. J. Ge, J. B. Chen, D. X. Liu, W. W. Wang, Z. H. Yang, W. Qi, C. Tian, Z. Q. Yuan, B. Zhang, L. Yang, J. L. Jiao, B. Cui, W. M. Zhou, L. F. Cao, C. T. Zhou, Y. Q. Gu,
B. H. Zhang, S. P. Zhu, and X. T. He, Phys. Rev. Lett. 120, 195001 (2018).

44. W. Fan, Y. Jiang, J. Wang, X. Wang, G. Y. Li, H. Wei, D. Huang, X. Lu, X. Pan, Z. Qiao, C. Wang, H. Chen, P. Zhang, F. H. Wen, Z. L. Xiao, S. J. Zhang, X. C. Li, J. Zhu, and Z. Lin, High Power Laser Sci. Eng. 6, e34 (2018).

45. L. Hu, S. Chen, J. Tang, B. Wang, T. Meng, W. Chen, L. Wen, J. Hu, S. Li, Y. Xu, Y. Jiang, J. Zhang, and Z. Jiang, High Power Laser Sci. Eng. 2, e1 (2014).

46. J. Zhang, D. Wu, J. Zheng, K. Zheng, Q. Zhu, and X. Zhang, Proc. SPIE 9294, 92940N (2014).

47. J. Shao, K. Yi, and M. Zhu, Proc. SPIE 9983, 998308 (2016).

48. A. Guo, H. Zhu, Z. Yang, S. Tang, X. Xie, and J. Zhu, Acta Opt. Sin. 33, 0311001 (2013).

49. W. Jiang, Y. Zhang, C. Rao, N. Ling, C. Guan, M. Li, Z. Yang, and G. Shi, Acta Opt. Sin. 31, 0901006 (2011).

50. D. Zhao, L. Wan, Z. Lin, P. Shao, and J. Zhu, High Power Laser Sci. Eng. 3, e7 (2015).

51. X. Ouyang, Y. Cui, J. Zhu, B. Zhu, and J. Zhu, Appl. Opt. 55, 7538 (2016).

52. C. Liu, L. Ji, L. Yang, D. Zhao, Y. Zhang, D. Liu, B. Zhu, and Z. Lin, Appl. Opt. 55, 2800 (2016).

53. Q. Tang, L. Yang, Y. Guo, X. Ouyang, S. Tang, and B. Zhu, Chin. J. Lasers 40, 0905006 (2013).

54. P. Zeng, D. Liu, Z. Peng, X. Tang, T. Ji, B. Zhu, D. Liu, and J. Zhu, Chin. J. Lasers 41, 0302009 (2014).

55. P. Zeng, X. Tang, X. Yang, T. Ji, M. Jiang, B. Zhu, J. Zhu, D. Liu, and L. Shen, High Power Laser Part. Beams 24, 2595 (2012).

56. Y. Gao, Y. Cui, H. Li, L. Gong, Q. Lin, D. Liu, B. Zhu, W. Ma, J. Zhu, and Z. Lin, Opt. Laser Technol. 100, 87 (2018).

57. Y. Y. Wang, Y. Q. Gao, H. D. Zhu, X. H. Lu, J. Y. Zhang, Y. J. Guo, H. C. Hui, and B. Q. Zhu, Acta Phys. Sin. 62, 055201 (2013).

58. W. F. Huang, J. F. Wang, X. H. Lu, and X. C. Li, Laser Phys. 23, 035804 (2013).

59. Z. Ren, J. Zhu, Z. Liu, and X. Yang, High Power Laser Sci. Eng. 6, e1 (2018).

60. B. Wang, J. Zhang, S. Shi, K. You, and J. Zhu, High Power Laser Sci. Eng. 4, e9 (2016).

61. J. Zhang, Y. Zhang, W. Ma, J. Zhu, D. A. Liu, L. Yang, J. Zhu, and Z. Lin, Acta Opt. Sin. 35, 0414004 (2015).

62. Y. Li, J. Zhu, Y. Wu, X. Jiao, and X. Pang, Proc. SPIE 9621, 96210Z (2015).

63. Z. Ren and J. Zhu, Proc. SPIE 8551, 855109 (2012).

64. F. Liu, J. Zhu, J. Xu, Q. Shan, K. Xiao, and X. Zhang, Chin. Opt. Lett. 10, 041402 (2012).

65. Z. Jiao, P. Shao, D. Zhao, R. Wu, L. Ji, L. Wang, L. Xia, D. Liu, Y. Zhou, L. Ju, Z. Cai, Q. Ye, Z. Qiao, N. Hua, Q. Li, W. Pan, L. Ren, M. Sun, J. Zhu, and Z. Lin, High Power Laser Sci. Eng. 6, e14 (2018).

66. D. T. Casey, J. L. Milovich, V. A. Smalyuk, D. S. Clark, H. F. Robey, A. Pak, A. G. MacPhee, K. L. Baker, C. R. Weber, T. Ma, H.-S. Park, T. Döppner, D. A. Callahan, S. W. Haan, P. K. Patel, J. L. Peterson, D. Hoover, A. Nikroo, C. B. Yeamans, F. E. Merrill, P. L. Volegov, D. N. Fittinghoff, G. P. Grim, M. J. Edwards, O. L. Landen, K. N. Lafortune, B. J. MacGowan, C. C. Widmayer, D. B. Sayre, R. Hatarik, E. J. Bond, S. R. Nagel, L. R. Benedetti, N. Izumi, S. Khan, B. Bachmann, B. K. Spears, C. J. Cerjan, M. Gatu Johnson, and J. A. Frenje, Phys. Rev. Lett. 115, 105001 (2012).

67. Y. Zhang, G. Li, W. Fan, X. Wang, Y. Jiang, and Z. Shi, Laser Optoelectron. Prog. 53, 081405 (2016).

68. D. He, S. Kang, L. Zhang, L. Chen, Y. Ding, Q. Yin, and L. $\mathrm{Hu}$, High Power Laser Sci. Eng. 5, e1 (2017). 
69. G. W. Zhang, X. Q. Lu, H. B. Cao, X. H. Yin, F. N. Lu, Z. Zhang, J. H. Li, R. G. Wang, W. X. Ma, and J. Zhu, Acta Phys. Sin. 61, 024201 (2012).

70. P. Zhang, Y. Jiang, J. Wang, W. Fan, X. Li, and J. Zhu, High Power Laser Sci. Eng. 5, e23 (2017).

71. C. Wang, H. Wei, Y. Jiang, J. Wang, Z. Qiao, J. Guo, W. Fan, and X. Li, Chin. Opt. Lett. 14, 121402 (2016).

72. J. F. Wang, X. C. Li, H. Wei, and J. Q. Zhu, Chin. Phys. Lett. 25, 524 (2008).

73. D. Huang, W. Fan, X. Li, and Z. Lin, Proc. SPIE 8556, 855615 (2012).

74. D. Z. Liu, J. Q. Zhu, R. F. Xu, and D. Y. Fan, Opt. Eng. 43, 2066 (2004).

75. S. Tang, Y. Guo, D. Liu, L. Yang, X. Jiang, Z. Peng, and B. Zhu, High Power Laser Sci. Eng. 6, e5 (2018).

76. X. Pan, S. P. Veetil, C. Liu, H. Tao, Y. Jiang, Q. Lin, X. Li, and J. Zhu, Laser Phys. Lett. 13, 055001 (2016).

77. H. Wang, C. Liu, X. He, X. Pan, S. Zhou, R. Wu, and J. Zhu, High Power Laser Sci. Eng. 2, e25 (2014).

78. H. Tao, S. P. Veetil, X. Pan, C. Liu, and J. Zhu, Chin. Opt. Lett. 14, 071203 (2016)
79. D. Zhao, R. Wu, Z. Lin, L. Wang, and J. Zhu, Proc. SPIE 9237, 92371V (2014).

80. Y. Guo, S. Tang, X. Jiang, Y. Peng, B. Zhu, and Z. Lin, High Power Laser Sci. Eng. 2, e21 (2014).

81. H. Hui, J. Zhang, X. Lu, J. Zhang, X. Chen, X. Jiang, B. Zhu, and Z. Lin, Chin. Opt. Lett. 14, 031405 (2016).

82. Y. F. Hao, M. Y. Sun, Y. J. Guo, S. Shi, X. Pan, X. Y. Pang, and J. Q. Zhu, Opt. Express 26, 8791 (2018).

83. L. Ren, P. Shao, D. Zhao, Y. Zhou, Z. Cai, N. Hua, Z. Jiao, L. Xia, Z. Qiao, R. Wu, L. Ji, D. Liu, L. Ju, W. Pan, Q. Li, Q. Ye, M. Sun, J. Zhu, and Z. Lin, High Power Laser Sci. Eng. 6, e10 (2018).

84. D. Zhang, L. Qian, P. Yuan, H. Zhu, S. Wen, and C. Xu, Opt. Lett. 33, 1969 (2008).

85. J. Zhu, X. Xie, M. Sun, J. Kang, Q. Yang, A. Guo, H. Zhu, P. Zhu, Q. Gao, X. Liang, Z. Cui, S. Yang, and Z. Lin, High Power Laser Sci. Eng. 6, e29 (2018).

86. P. Zhu, X. L. Xie, J. Kang, Q. W. Yang, H. D. Zhu, A. L. Guo, M. Z. Sun, Q. Gao, Z. R. Cui, X. Liang, S. H. Yang, D. J. Zhang, and J. Q. Zhu, High Power Laser Sci. Eng. 6, e8 (2018). 